\title{
Seismic Attenuation for Reservoir Characterization DE-FC26-01BC15356
}

\author{
Quarterly Report \\ Jan. 1 - Mar. 31, 2002
}

Issued April, 2002

\author{
Contributors \\ Dr. Joel Walls* \\ Dr. M. T. Taner* \\ Dr. Gary Mavko** \\ Dr. Jack Dvorkin**
}

*Principal Contractor:

Rock Solid Images

2600 S. Gessner Suite 650

Houston, TX, 77063

**Subcontractor:

Petrophysical Consulting Inc.

730 Glenmere Way

Emerald Hills, CA, 94062 


\section{Disclaimer}

This report was prepared as an account of work sponsored by the United States Government. Neither the United States Government nor any agency thereof, nor any of their employees, makes any warranty, expressed or implied, or assumes any legal liability or responsibility for the accuracy, completeness, or usefulness of any information, apparatus, product, or process disclosed, or represents that its use would not infringe privately owned rights. Reference herein to any specific commercial poduct, process, or service by trade name, trademark, manufacturer, or otherwise does not necessarily constitute or imply its endorsement, recommendation, or favoring by the United States Government or any agency thereof. The views and opinions of authors expressed herein do not necessarily state or reflect those of the United States Government or any agency thereof. 


\section{Contents}

SECTION 1: ATTENUATION WITH PATCHY SATURATION ……………………………………...

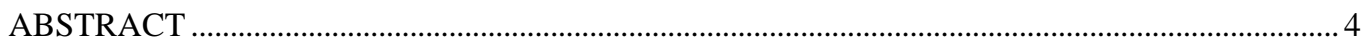

UPPER AND LOWER ELASTIC LIMITS AT PARTIAL SATURATION ...................................... 4

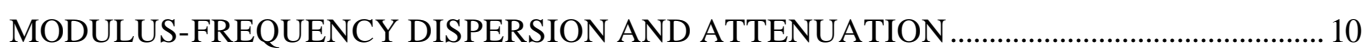

ATTENUATION AND INVERSE QUALITY FACTOR ……………………………………..... 12

ADDING ATTENUATION FROM SEPARATE MECHANISMS..................................................... 13

ATTENUATION AT PARTIAL SATURATION - SYNTHETIC EXAMPLES …………………... 14

MORE SYNTHETIC EXAMPLES - VERY SOFT SANDS ............................................................. 17

EFFECTS OF DRY FRAME MODULUS AND SATURATION .................................................. 18

SYNTHETIC EXAMPLES BASED ON WELL LOG DATA ……………………………………........ 19

APPLICABILITY OF THE MODEL - DATA AND SPECULATIONS ………………………..... 24

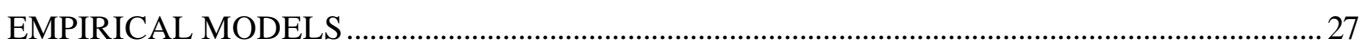

COMBINING KOM AND PATCHY MODEL ………………………………………………....2

SANDWICH MODELS FOR SYNTHETIC SEISMIC MODELING.............................................. 30

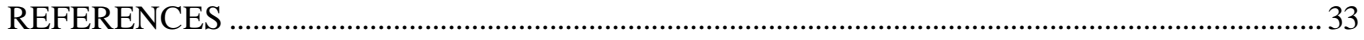

SECTION 2: Q, ABSORPTION AND DISPERSION BY SEVERAL DIFFERENT METHODS ........ 34

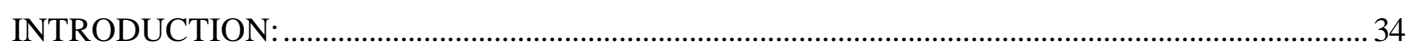

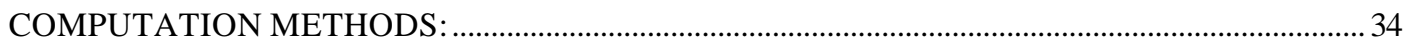

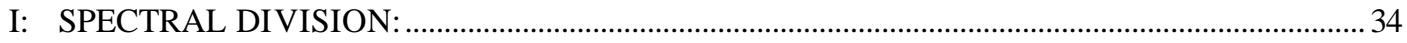

II: THE RISE TIME METHOD:

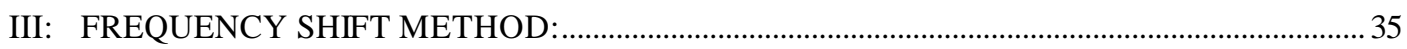

IV: MINIMUM PHASE INVERSE SYNTHETIC DIVISION METHOD:...........................................

V: SECOND METHOD FOR SYNTHETIC DIVISION: ADAPTIVE COMPUTATION: ..............38

VI: RELATIVE Q FROM COMPLEX TRACE ATTRIBUTES:.............................................................. 39

VII: RELATIVE Q FROM COMPLEX TRACE ATTRIBUTES: ........................................................ 40

VIII: RELATIVE Q FROM WAVELET WIDTH-VS.-BAND WIDTH RELATION:...................... 41

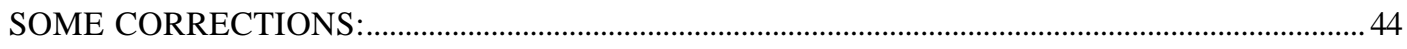

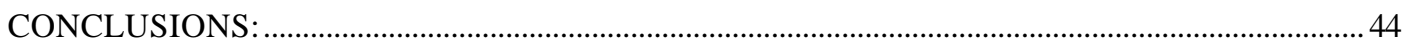

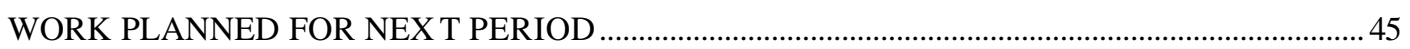

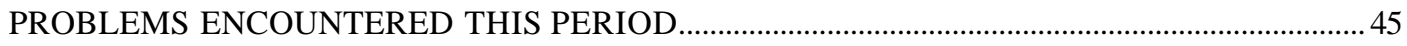




\title{
SECTION 1: ATTENUATION WITH PATCHY SATURATION
}

\begin{abstract}
Wave-induced variations of pore pressure in a partially-saturated reservoir result in oscillatory liquid flow. The viscous losses during this flow are responsible for wave attenuation. The same viscous effects determine the changes in the dynamic bulk modulus of the system versus frequency. These changes are necessarily linked to attenuation via the causality condition. We analytically quantify the frequency dependence of the bulk modulus of a partially saturated rock by assuming that saturation is patchy and then link these changes to the inverse quality factor. As a result, the Pwave attenuation is quantitatively linked to saturation and thus can serve as a saturation indicator.
\end{abstract}

\section{UPPER AND LOWER ELASTIC LIMITS AT PARTIAL SATURATION}

The frequency range of seismic radiation used for illuminating the subsurface spans four orders of magnitude, from $10^{1}$ (seismic) to $10^{4}$ (sonic logging) Hz. Typically, the pore-scale Biot's and squirt flow attenuation mechanisms are not engaged at these frequencies. Still, viscoelastic effects and attenuation may arise from the oscillatory liquid cross-flow between fully-liquid-saturated patches and the surrounding rock with gas. The main condition for fluid-related attenuation in the practical frequency range is the existence of such patches whose length scale is several orders of magnitude larger than the pore scale (Figure 1).

To understand physical reasons for the existence of patchy saturation, let us consider a volume of rock that consists of several sand patches with clay content slightly varying among them. These clay-content variations may have a small effect on the dry-frame elastic moduli but dramatically affect permeability (e.g., Yin, 1993) and, therefore, capillary pressure curves. Then, in a state of capillary equilibrium, this elastically homogeneous volume may have a patchy saturation pattern (Knight et al., 1998). Indeed, if capillary pressure is the same for the adjacent patches whose capillary pressure curves are different, these patches can have very different fluid saturation. Visual proof that patches form in oil-water and air-water systems in the laboratory has been presented by Chatenever and Calhoun (1952) and Cadoret (1993). Indirect evidence that patches exist 
in situ has been presented by Brie et al. (1995) and Dvorkin et al. (1999).
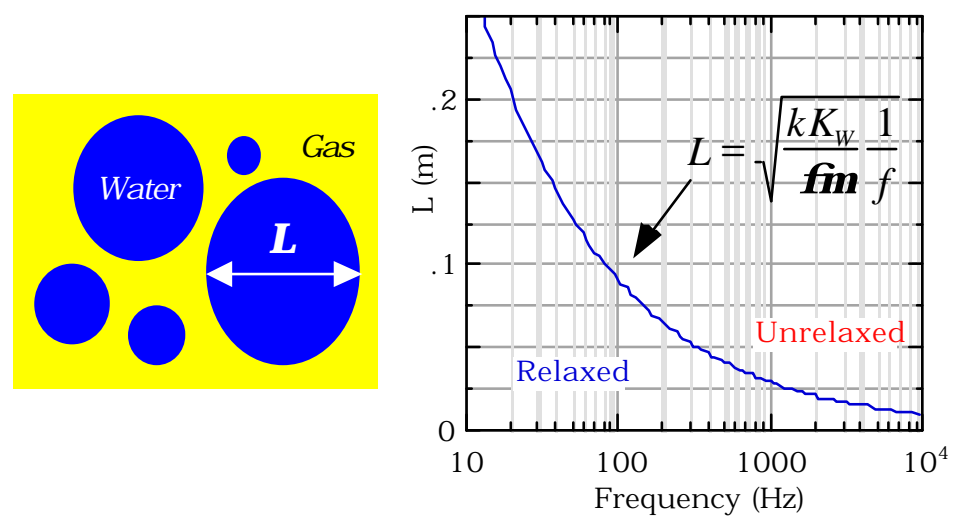

Figure 1. Left -- Macroscopic fully-saturated patches in a partially-saturated reservoir. Right - Critical length versus frequency for a patch saturated with water with the bulk modulus of $2.5 \mathrm{GPa}$ and viscosity $1 \mathrm{cPs}$. The permeability of the patch is $100 \mathrm{mD}$ and porosity is 0.3 .

The size $L$ of a continuous patch occupied solely by the liquid phase determines the effective bulk modulus of the partially saturated rock at a fixed global saturation and frequency. If the patch is small so that, $L<\sqrt{D / f}\left(D=k K_{W} / \phi \mu\right.$ is the diffusivity; $k$ is the permeability; $K_{W}$ is the bulk modulus of the liquid; $\phi$ is the porosity; and $\mu$ is the dynamic viscosity of the liquid), or the frequency is small, the wave-induced pore pressure variations between the patch and surrounding gas-saturated rock equilibrate during the wave period. The patch is relaxed. This critical size $L$ for high-porosity highpermeability rock is plotted versus frequency $f$ in Figure 1. It appears that the critical size may be as small as $10 \mathrm{~cm}$ at a frequency of $50 \mathrm{~Hz}$. This length is approximately the scale of heterogeneity in the reservoir required for viscoelastic effects and attenuation to become detectable at seismic frequency.

If the patch size is smaller than $L$, the pressure oscillations in the patch will quickly equilibrate with the surrounding gas-saturated region. As a result, the entire partially saturated region can be treated as homogeneously saturated with a liquid-gas mixture whose bulk modulus $K_{F}$ is the Reuss average of those of the liquid $\left(K_{W}\right)$ and gas $\left(K_{G}\right)$ :

$$
\frac{1}{K_{F}}=\frac{S_{W}}{K_{W}}+\frac{1-S_{W}}{K_{G}}
$$


where $S_{W}$ is liquid (water) saturation.

Then the bulk modulus of the partially saturated region $K_{\text {Sat } 0}$ is determined by Gassmann's equation

$$
K_{\text {Sat } 0}=K_{S} \frac{\phi K_{D r y}-(1+\phi) K_{F} K_{D r y} / K_{S}+K_{F}}{(1-\phi) K_{F}+\phi K_{S}-K_{F} K_{D r y} / K_{S}},
$$

where $K_{D r y}$ is the bulk modulus of the dry frame of the rock; $K_{S}$ is the bulk modulus of the mineral phase; and $\phi$ is the porosity. The shear modulus of the partially saturated region $G_{S a t}$ is the same as that of the dry-rock frame $G_{D r y}$.

If the patch is large, $L>\sqrt{D / f}$, or the frequency is high, the wave-induced pore pressure variations in it will not equilibrate with the surrounding dry region during the wave period. The patch in unrelaxed. Then the bulk modulus of the patch $K_{P}$ will appear to be that of the fully liquid saturated rock:

$$
K_{P}=K_{S} \frac{\phi K_{D r y}-(1+\phi) K_{W} K_{D r y} / K_{S}+K_{W}}{(1-\phi) K_{W}+\phi K_{S}-K_{W} K_{D r y} / K_{S}} .
$$

Then the volumetric concentration of the fully saturated patches in the system is $S_{W}$ and the effective bulk modulus of the partially saturated region $K_{\text {Sato }}$ is determined by the constant-shear-modulus equation

$$
\frac{1}{K_{\text {Sato }}+(4 / 3) G_{D r y}}=\frac{S_{W}}{K_{P}+(4 / 3) G_{D r y}}+\frac{1-S_{W}}{K_{S_{W}=0}+(4 / 3) G_{D r y}}
$$

where $K_{S_{W}=0}$ is the bulk modulus of the gas-saturated rock given by Gassmann's equation

$$
K_{S_{W}=0}=K_{S} \frac{\phi K_{D r y}-(1+\phi) K_{G} K_{D r y} / K_{S}+K_{G}}{(1-\phi) K_{G}+\phi K_{S}-K_{G} K_{D r y} / K_{S}} .
$$

$K_{S_{W}=0}$ is very close to $K_{D r y}$ due to the high compressibility of gas.

If the size of the patch is fixed and the frequency is changing, the effective bulk modulus of partially saturated rock will vary between its low-frequency limit $K_{\text {Sat } 0}$ and 
high-frequency limit $K_{\text {Sato }}$. The effective compressional modulus $M_{\text {Sat }}$ will vary between $M_{\text {Sat } 0}$ and $M_{\text {Sato }}$ :

$$
K_{S a t 0}+\frac{4}{3} G_{D r y}=M_{S a t 0} \leq M_{S a t} \leq M_{S a t \infty}=K_{S a t \infty}+\frac{4}{3} G_{D r y}
$$

The same approach can be used to describe the low- and high-frequency behavior of a patchy system if only P-wave data are available. The P-only fluid substitution equation of Mavko et al. (1995) is essentially Gassmann's equation where the bulk moduli of the rock and the solid phase are replaced by their compressional moduli. The compressional modulus is the product of the bulk density and P-wave velocity squared. Specifically, the low-frequency compressional modulus $M_{\text {Sat } 0}$ is

$$
M_{\text {Sat } 0}=M_{S} \frac{\phi M_{D r y}-(1+\phi) K_{F} M_{D r y} / M_{S}+K_{F}}{(1-\phi) K_{F}+\phi M_{S}-K_{F} M_{D r y} / M_{S}}
$$

where $M_{D r y}$ is the compressional modulus of the dry frame of the rock; and $M_{S}$ is the compressional modulus of the mineral phase.

The high-frequency limit $M_{\text {Sato }}$ is given by Equation (4) which we re-write below as

$$
\frac{1}{M_{\text {Sato }}}=\frac{S_{W}}{M_{P}}+\frac{1-S_{W}}{M_{S_{W}=0}}
$$

where

$$
M_{P}=M_{S} \frac{\phi M_{D r y}-(1+\phi) K_{W} M_{D r y} / M_{S}+K_{W}}{(1-\phi) M_{W}+\phi M_{S}-K_{W} M_{D r y} / M_{S}}
$$

and

$$
M_{S_{W}=0}=M_{S} \frac{\phi M_{D r y}-(1+\phi) K_{G} M_{D r y} / M_{S}+K_{G}}{(1-\phi) K_{G}+\phi M_{S}-K_{G} M_{D r y} / M_{S}} .
$$

$M_{S_{W}=0}$ is very close to $M_{D r y}$ due to the high compressibility of gas.

An example of the low- and high-frequency bounds for the compressional modulus of 
a partially saturated rock is given in Figure 2. The difference between $M_{\text {Sat } 0}$ and $M_{\text {Satoo }}$ is large at partial saturation, especially in the low-gas-saturation domain.

It appears in Figure 2 that the curve described by Equation (8) is close to a straight line that connects the zero-saturation and full-saturation points. This means that $M_{\text {Sato }}$ can be approximately modeled as a result of using a fictitious fluid whose bulk modulus is a linear combination of those of gas and water,

$$
K_{F P}=S_{W} K_{W}+\left(1-S_{W}\right) K_{G},
$$

in the fluid substitution equations (2) or (7). Equation (9) does not describe a real physical entity resulting from mixing two pore fluid phases. It is simply a convenient approximate (and physically deceptive) way of conducting patchy fluid substitution.
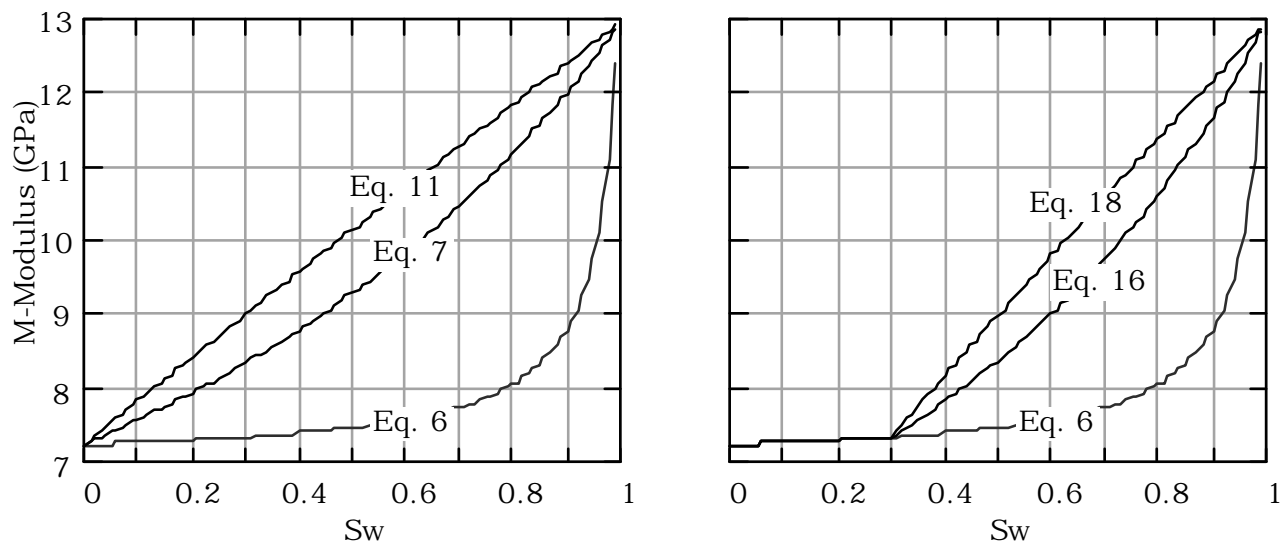

Figure 2. Left -- The compressional modulus versus saturation in sand of porosity 0.35 , where the dry-frame compressional modulus is $7 \mathrm{GPa}$. The compressional modulus of the mineral phase is $100 \mathrm{GPa}$, and those of water and gas are 2.5 and 0.1 GPa, respectively. The three curves have been calculated using Equations (6), (7), and (9), as indicated on the curves. The irreducible water saturation is zero. Right-Same with irreducible water saturation 0.3. Equations used are indicated on the curves.

In Figure 2, left, the range of water saturation is between 0 and $1 . \quad$ In reality, there is always irreducible water saturation in a gas reservoir. This simply means that patchy saturation pattern cannot exist at $S_{W}<S_{W I r r}$ and the deviation of the patchy saturation curve from the homogeneous saturation curve starts at some finite water saturation (Figure 2, right).

Consider a porous system where water saturation is at its minimum value $S_{W I r r}$. Let 
us add some water to this system to arrive at the global water saturation $S_{W}>S_{W I r r}$. Let us also assume that all additional water is concentrated in fully saturated patches that start to appear as soon as $S_{W}$ exceeds $S_{W I r}$. Then the volumetric concentration $f_{P}$ of these patches in the partially saturated system is

$$
f_{P}=\frac{S_{W}-S_{W I r r}}{1-S_{W I r r}}
$$

The bulk modulus of the fully saturated patch is given by Equation (3). The bulk modulus $K_{S_{W}=S_{W I r}}$ of the surrounding rock at $S_{W}=S_{W I r r}$ is given by Gassmann's equation

$$
K_{S_{W}=S_{W I r r}}=K_{S} \frac{\phi K_{D r y}-(1+\phi) K_{F I r r} K_{D r y} / K_{S}+K_{F I r r}}{(1-\phi) K_{F I r r}+\phi K_{S}-K_{F I r r} K_{D r y} / K_{S}},
$$

where $K_{F I r r}$ is the bulk modulus of the homogeneous water/gas mixture at $S_{W}=S_{W I r}$ :

$$
\frac{1}{K_{F I r r}}=\frac{S_{W I r r}}{K_{W}}+\frac{1-S_{W I r r}}{K_{G}}
$$

Then the bulk modulus of the system with patchy saturation at $S_{W}>S_{W I r r}$ is given by the constant-shear-modulus equation

$$
\begin{aligned}
& \frac{1}{K_{\text {SatIrroo }}+(4 / 3) G_{D r y}}=\frac{f_{P}}{K_{P}+(4 / 3) G_{D r y}}+\frac{1-f_{P}}{K_{S_{W}=S_{W I r r}}+(4 / 3) G_{D r y}} \\
& =\frac{\left(S_{W}-S_{W I r r}\right) /\left(1-S_{W I r r}\right)}{K_{P}+(4 / 3) G_{D r y}}+\frac{\left(1-S_{W}\right) /\left(1-S_{W I r r}\right)}{K_{S_{W}=S_{W I r r}}+(4 / 3) G_{D r y}} .
\end{aligned}
$$

The bulk modulus of the system at $S_{W}<S_{W I r r}$ is given by Equation (2).

The same approach can be used to calculate the compressional modulus of the system by the approximate P-only fluid substitution. The compressional modulus at $S_{W}<S_{W I r r}$ is given by Equation (7). For $S_{W}>S_{W I r r}$, the compressional modulus $M_{\text {SatIrroo }}$ in the system with patchy saturation is given by Equation (15) which we re-write here as

$$
\frac{1}{M_{\text {SatIrroo }}}=\frac{\left(S_{W}-S_{W I r r}\right) /\left(1-S_{W I r r}\right)}{M_{P}}+\frac{\left(1-S_{W}\right) /\left(1-S_{W I r r}\right)}{M_{S_{W}=S_{W I r r}}},
$$


where $M_{P}$ comes from Equation (9) and $M_{S_{W}=S_{W I r}}$ is

$$
M_{S_{W}=S_{W I r r}}=M_{S} \frac{\phi M_{D r y}-(1+\phi) K_{F I r r} M_{D r y} / M_{S}+K_{F I r r}}{(1-\phi) K_{F I r r}+\phi M_{S}-K_{F I r r} M_{D r y} / M_{S}},
$$

with $K_{F I r r}$ given by Equation (14).

The resulting compressional modulus versus saturation curve shown in Figure 2, right, can be approximated by a line resulting from substituting a fictitious fluid mix into Gassmann's equation or the P-only fluid substitution equation for the compressional modulus. The bulk modulus of this fictitious mix is given by Equation (1) for $S_{W}<S_{W I r r}$ and by

$$
K_{F P I r r}=\frac{S_{W}-S_{W I r r}}{1-S_{W I r r}} K_{W}+\frac{1-S_{W}}{1-S_{W I r r}} K_{F I r r}
$$

for $S_{W}>S_{W I r r}$, where $K_{F I r r}$ is the bulk modulus of the homogeneous gas/water mix at $S_{W}=S_{W I r r}$, as given by Equation (14).

\section{MODULUS-FREQUENCY DISPERSION AND ATTENUATION}

The difference between the elastic moduli calculated using the homogeneous and patchy saturation equations is, in essence, the difference between the low-frequency and high-frequency values of the elastic moduli. The effect of the apparent elastic moduli increase with increasing frequency is called the modulus-frequency dispersion (or the velocity-frequency dispersion, if we consider the elastic-wave velocity instead of the elastic moduli). Let us reiterate that here we examine the modulus-frequency dispersion in the practical frequency range that includes seismic and sonic frequencies. As a result, we do not consider the ultrasonic frequency dispersion effects related to the microscopic squirt flow and the Biot mechanisms.

The transition between the lower (homogeneous saturation) curve and the upper (patchy) curve in Figure 2 is the transition between the low and high frequency. The corresponding elastic modulus dispersion is necessarily accompanied by absorption, 
according to the Kramer-Kronig relation for linear viscoelastic systems. To quantify this absorption, one needs to identify the frequency variation range (e.g., the low-frequency limit is at $10 \mathrm{~Hz}$ and the high-frequency limit is at $10 \mathrm{kHz}$ ) and also to know how the elastic modulus evolves as it changes between its low-frequency and its high-frequency values.

Two basic viscoelastic models give examples of linking attenuation to modulusfrequency dispersion. According to the standard Inear solid (SLS) model, the elastic modulus $M$ is related to frequency $f$ as

$$
M(f)=\frac{M_{0} M_{\infty}\left[1+\left(f / f_{C R}\right)^{2}\right]}{M_{\infty}+M_{0}\left(f / f_{C R}\right)^{2}},
$$

where $M_{0}$ is the low-frequency limit; $M_{\infty}$ is the high-frequency limit; and $f_{C R}$ is the critical frequency at which the transition occurs from the low-frequency to the highfrequency limit. The corresponding inverse quality factor is

$$
\frac{1}{Q}=\frac{\left(M_{\infty}-M_{0}\right)\left(f / f_{C R}\right)}{\sqrt{M_{0} M_{\infty}}\left[1+\left(f / f_{C R}\right)^{2}\right]} .
$$

The maximum attenuation occurs at $f=f_{C R}$ and is

$$
\left(\frac{1}{Q}\right)_{\max }=\frac{M_{\infty}-M_{0}}{2 \cdot \sqrt{M_{0} M_{\infty}}} .
$$

In the example shown in Figure 2, right, $M_{0}$ is determined by the lower curve and is about $8 \mathrm{GPa}$ at water saturation 0.7. The patchy saturation curve drawn according to Equation (16) determines $M_{\infty}$ which is about $10 \mathrm{GPa}$ at water saturation 0.7. By selecting $f_{C R}=500 \mathrm{~Hz}$ and using Equations (19) and (20), we can determine the shape of the modulus-porosity and attenuation-frequency curves (Figure 3, left). The maximum inverse quality factor is about 0.11 .

The constant (or nearly constant) $Q(\mathrm{CQ})$ model assumes that the quality factor is constant within a frequency range. Then the inverse quality factor is 


$$
\frac{1}{Q}=\frac{\pi}{\log \left(f_{1} / f_{0}\right)} \frac{M_{1}-M_{0}}{2 M_{0}}
$$

where $M_{0}$ is the modulus at frequency $f_{0}$ and $M_{1}$ is the modulus at frequency $f_{1}$. It follows from equation (22) that the modulus changes proportionally to the logarithm of frequency, i.e.,

$$
M=M_{0}\left(\frac{2}{\pi Q} \log \frac{f}{f_{0}}+1\right) .
$$

By selecting from Figure 2, right, $M_{0}=8 \mathrm{GPa}$ and $M_{1}=10 \mathrm{GPa}$, and assuming that $f_{0}=1 \mathrm{~Hz}$ and $f_{1}=10 \mathrm{kHz}$, we find, according to Equation (22), that the inverse quality factor is about 0.098. Fortunately, this value is not very sensitive to the selection of the low and high frequency values. For example, if we selected $f_{0}=10 \mathrm{~Hz}$ and $f_{1}=20$ $\mathrm{kHz}$, the resulting inverse quality factor would be about 0.12 .

It is also encouraging that the inverse quality values determined from the two viscoelastic models (SLS and CQ) are about the same.
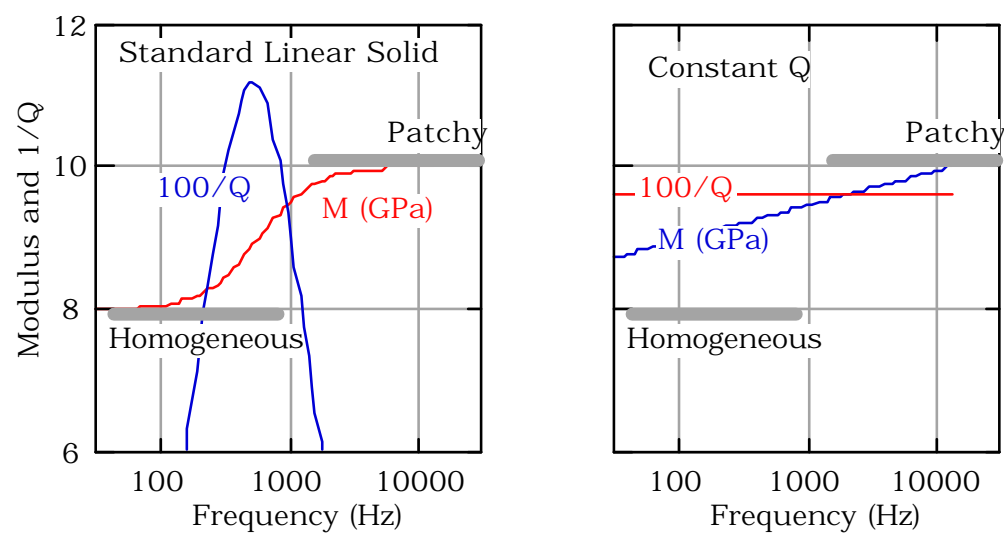

Figure 3. The compressional modulus and inverse quality factor versus frequency for the low-frequency modulus value $8 \mathrm{GPa}$ and high-frequency modulus value 10 GPa. Left - SLS model. Right - CQ model.

\section{ATTENUATION AND INVERSE QUALITY FACTOR - DEFINITIONS AND RELATIONS}

The attenuation coefficient $\alpha$ is defined as the exponential decay coefficient of a harmonic wave: 


$$
A(x, t)=A_{0} \exp [-\alpha(\omega) x] \exp [i(\omega t-k x)]
$$

where $A$ is the amplitude of the signal; $A_{0}$ is the initial amplitude; $t$ is time; $x$ is the spatial coordinate; $\omega=2 \pi f$ is the angular frequency; and $k$ is the wavenumber. The attenuation coefficient is related to the inverse quality factor as

$$
\alpha=Q^{-1} \pi f / V=\pi /(Q T V)=\pi /(Q \lambda)
$$

where $V$ is the phase velocity; $T$ is the period; and $\lambda$ is the wavelength. By substituting Equation (25) into Equation (24) we obtain:

$$
\frac{A(x, t)}{A_{0}}=\exp \left[-\frac{\pi}{Q} \frac{x}{\lambda}\right] \exp [i(\omega t-k x)]
$$

Let us ask ourselves a question - over how many wavelengths will the amplitude decrease $10^{\mathrm{n}}$ times? The answer comes from Equation (26):

$$
\exp \left[-\frac{\pi}{Q} \frac{x}{\lambda}\right]=10^{-n} \Rightarrow \frac{x}{\lambda}=n \frac{2.3}{\pi} Q=0.733 n Q
$$

which means that the required number of wavelengths is $0.733 n Q$.

Sometimes $\alpha$ is given in $\mathrm{dB}$ per unit length. The conversion coefficient is 8.686 , i.e.,

$$
\left.\alpha_{\text {dB }} / \text { Length } \quad\right]=8.686 Q^{-1} \pi f / V \text {. }
$$

\section{ADDING ATTENUATION FROM SEPARATE MECHANISMS}

In our attempts to mathematically relate the elastic wave attenuation to reservoir properties and conditions, we will certainly have to decompose the phenomenon, in its entity, into separate mechanisms that are described by different mathematical models. This mathematical decomposition may be a reflection of a physically realizable decomposition. For example, consider a situation where elastic waves attenuate in dry sandstone due to viscoelastic clay present in the frame. This is the first mechanism of attenuation. If this sandstone is partially saturated, an additional, patchy saturation dispersion, mechanism may be added to the first mechanism. The question is how to 
mathematically add these two attenuation mechanisms together to calculate the total attenuation resulting from the superposition of the two physical situations.

One simple assumption will be that the attenuation adds geometrically. In other words, if the first mechanism acts to reduce the initial amplitude of the signal $n$ times, from $A_{0}$ to $A_{1}=\exp \left(-\alpha_{1} x\right)$, over distance $x$, and the second mechanism independently acts to reduce the initial amplitude $m$ times, from $A_{0}$ to $A_{2}=\exp \left(-\alpha_{2} x\right)$, over the same distance, then, when acting together, the two mechanisms will reduce the initial amplitude, over the same distance, $n m$ times to

$$
A_{\text {Sum }}=n m A_{0}=\frac{A_{1}}{A_{0}} \frac{A_{2}}{A_{0}} A_{0}=A_{0} e^{-\alpha_{1} x} e^{-\alpha_{2} x}=A_{0} e^{-\left(\alpha_{1}+\alpha_{2}\right) x} .
$$

Therefore, attenuation coefficients and inverse quality factors calculated from separate mechanisms acting at the same time in the same reservoir can be added.

\section{ATTENUATION AT PARTIAL SATURATION - SYNTHETIC EXAMPLES}

Let us apply the patchy saturation modulus-frequency dispersion principle combined with SLS model to calculate attenuation in gas saturated samples. Our first example is for high-porosity unconsolidated sand. Specifically, we choose laboratory measurements of velocity on a hand-made mixture of Ottawa sand and kaolinite with $10 \%$ clay content (Yin, 1993). The room dry sample has porosity about $32 \%$ and bulk density of $1.8 \mathrm{~g} / \mathrm{cm}^{3}$. The P-wave velocity measured in the air-saturated sample is plotted versus the effective pressure in Figure 4a. The corresponding compressional modulus is shown in Figure 4b.
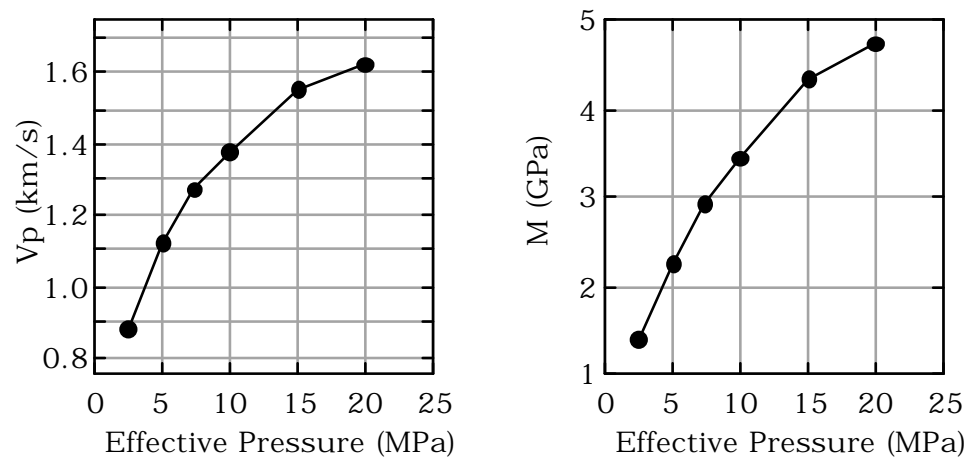

Figure 4. The P-wave velocity and corresponding compressional modulus versus effective pressure in a room-dry Ottawa sand with $10 \%$ kaolinite (experiments by Yin, 1993). 
In our attenuation calculations we assume that the irreducible water saturation is 0.1 and the bulk moduli of gas and water are 0.1 and $2.5 \mathrm{GPa}$, respectively. The inverse Pwave quality factor is plotted versus water saturation in Figure 5, left, for the effective pressure 20, 10, and $5 \mathrm{MPa}$. The corresponding attenuation coefficient in $\mathrm{dB} / \mathrm{m}$ is calculated from the inverse quality factor according to Equation (26) and plotted versus saturation in Figure 5, right. The frequency $f$ needed for the attenuation coefficient calculation was fixed at $5 \mathrm{kHz}$.

At a constant overburden, low effective pressure corresponds to high pore pressure. As a result, we can interpret the attenuation curves as corresponding to normally pressured gas sand (effective pressure $20 \mathrm{MPa}$ ); mildly overpressured sand (effective pressure $10 \mathrm{MPa}$ ) and strongly overpressured sand (effective pressure $5 \mathrm{MPa}$ ).

The first observation from Figure 5 is that using attenuation may help discriminate between large and small gas saturation - attenuation is maximum at very small gas saturation - and probably resolve the problem of "fizz" water, i.e., identify water-filled formations with only small amount of free gas present.

The second observation is that the $\mathrm{P}$-wave attenuation is only weakly affected by pore pressure. It may be hard to identify overpressured formations solely on the basis of $\mathrm{P}$ wave attenuation.
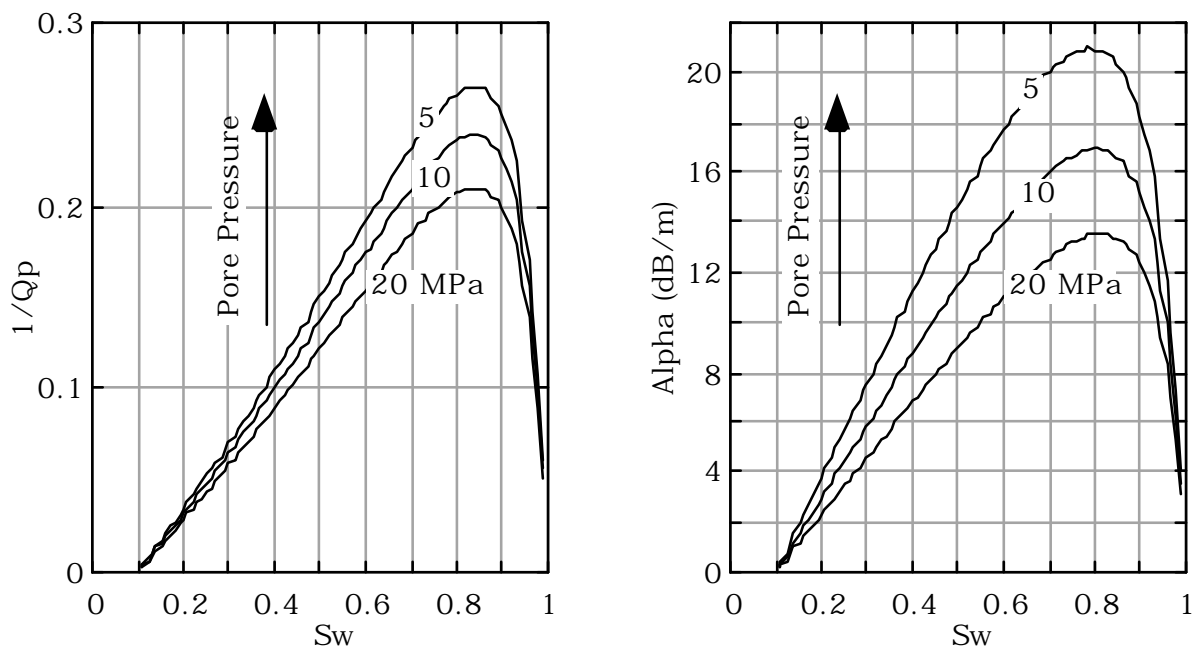

Figure 5. Left - the inverse quality factor versus water saturation in the sand/clay mixture (described in the text) at varying effective pressure (marked on the curves). Right -- the attenuation coefficient in $\mathrm{dB} / \mathrm{m}$. 
The second example is for low-to-medium porosity gas sandstone, similar to that described in Klimentos (1995). We choose porosity 13\%; dry-rock bulk density 2.3 $\mathrm{g} / \mathrm{cm}^{3}$; irreducible water saturation 0.15 ; and the dry-rock P-wave velocity $2.5 \mathrm{~km} / \mathrm{s}$. The corresponding compressional modulus is $14.4 \mathrm{GPa}$. The resulting inverse P-wave quality factor is plotted versus saturation in Figure 6, left and the attenuation coefficient in Figure 6, right. For the same situation but stiffer rock (the dry rock velocity $3.5 \mathrm{~km} / \mathrm{s}$ and the compressional modulus $28 \mathrm{GPa}$ ), the attenuation is even smaller but still noticeable.

As expected, the calculated attenuation in consolidated sandstone is smaller than that calculated in the soft sand. The attenuation coefficient values shown in Figure $6 \mathrm{~b}$ are smaller than those reported by Klimentos (1995) for a gas sandstone interval at about $30 \%$ water saturation ( $\alpha$ between 13 and $16 \mathrm{~dB} / \mathrm{m}$ ). One problem in matching the Klimentos (1995) measurements is that the exact parameters of his acquisition and rock properties have not been reported. In out attenuation coefficient calculations we assumed that the acquisition frequency was $5 \mathrm{kHz}$. If instead $10 \mathrm{kHz}$ was selected, the attenuation coefficient values would be twice those shown in Figure 6 and closer to those reported by Klimentos (1995).

The main lesson, however, is that we probably cannot predict exact absolute attenuation values from a mathematical model. The value of the modeling presented here is in its variational power -- the model probably cannot give us the background attenuation but instead can help us estimate attenuation changes due to saturation changes and other rock property and condition variations.
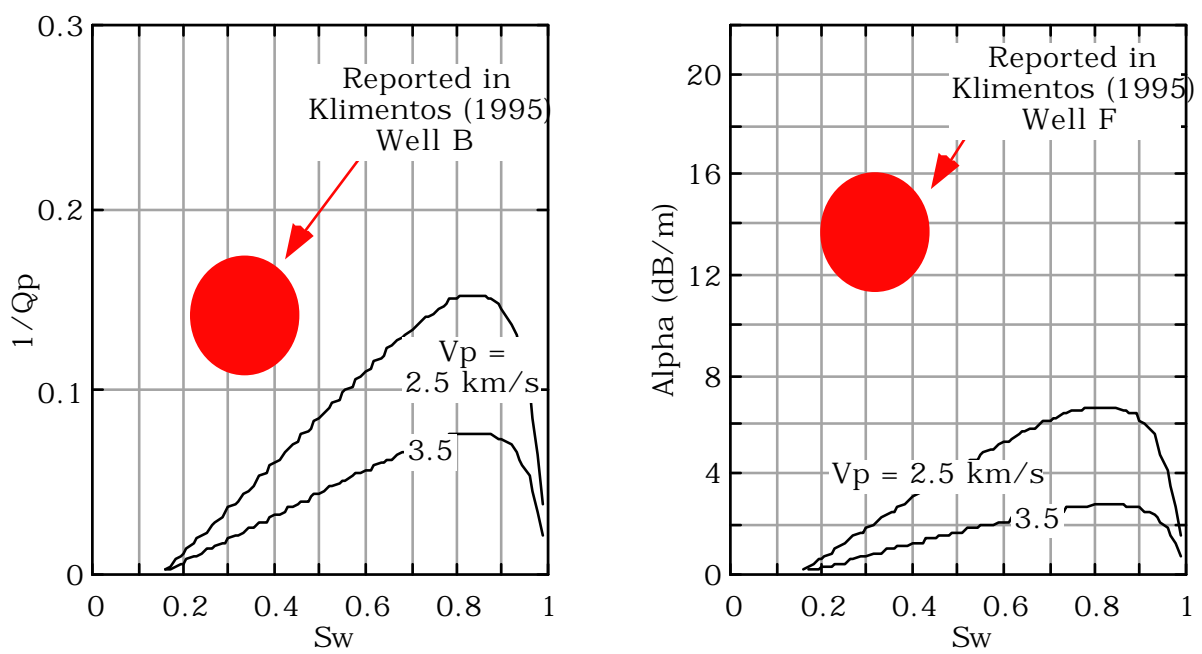

Figure 6. Left - the inverse quality factor versus water saturation in low-to- 
medium porosity gas sandstone described in the text. The upper curve is for the sandstone whose dry-rock P-wave velocity is $2.5 \mathrm{~km} / \mathrm{s}$. The lower curve is for stiffer sandstone with the dry-rock P-wave velocity $3.5 \mathrm{~km} / \mathrm{s}$. Right -- the attenuation coefficient in $\mathrm{dB} / \mathrm{m}$. The red circles show the range of values calculated from well log data and reported by Klimentos (1995) for two separate wells (B and F) within the gas zone with about $30 \%$ water saturation.

\section{MORE SYNTHETIC EXAMPLES - VERY SOFT SANDS}

Let us concentrate on applying the patchy saturation approach to modeling attenuation in very soft sands. In the following synthetic example we will use the same input values as in the example shown in Figure 5 except for the compressional modulus of the dry frame. We will systematically shift its value towards zero to mimic liquefaction (or rather "gasefaction") conditions which are probably present in severely overpressured geo-hazardous sands. Specifically, we choose three monotonically decreasing values of $M_{D r y}: 2 ; 0.5$; and $0.1 \mathrm{GPa}$. The resulting inverse quality factor is plotted versus water saturation in Figure 7, left. It exhibits a counterintuitive behavior: the softer the sand the smaller the P-wave attenuation. This result arises from the fact that the softer the sand the smaller the difference between the patchy and homogeneous elastic-modulus-versus-saturation curves (Figure 7, right). It is easy to show that for $M_{\text {Dry }}=0$ both curves are exactly the same.

The predicted effect probably reflects some aspects of a realistic physical situation. The most important lesson, however, is that for soft rocks one has to use exact patchy saturation equation such as Equations (15) and (16). The fictitious fluid approach reflected in Equations (11) and (18) may not be used in soft rocks because it will

mistakenly produce a large difference between the homogeneous and patchy saturation curves. 

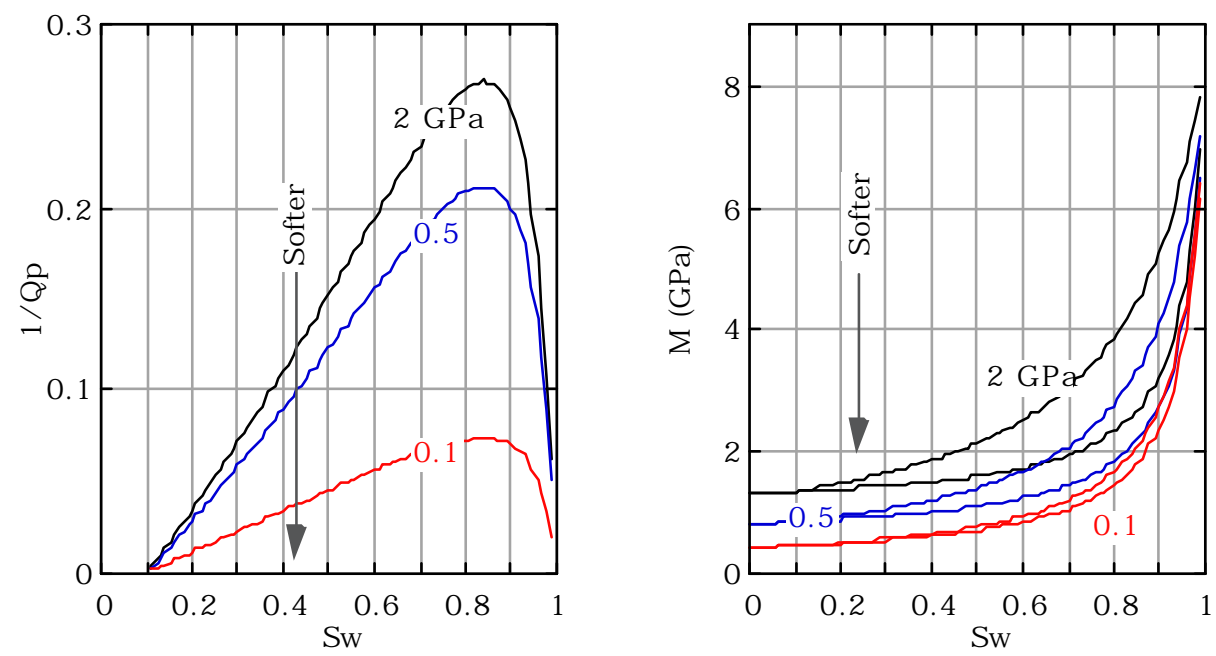

Figure 7. Left - the inverse quality factor versus water saturation in very soft sand. The calculation parameters are explained in the text. The curves are marked by the dry-frame compressional modulus values used and color-coded accordingly. Right -- the compressional modulus versus saturation for the same example. In each color group the upper curve is for patchy and the lower is for homogeneous saturation.

\section{EFFECTS OF DRY FRAME MODULUS AND SATURATION}

In the following synthetic examples we investigate the dependence of the P-wave attenuation on water sturation $\left(S_{W}\right)$ and the stiffness of the dry frame of the rock $\left(M_{D r y}\right)$. In the first example we examine very soft sand by gradually reducing $M_{D r y}$ from $3 \mathrm{GPa}$ to zero. This modulus reduction may reflect an increase in pore pressure from a high to a very high value approaching the overburden. The porosity of the sand was assumed constant 0.4. The irreducible water saturation was fixed at 0.05 . The bulk moduli of water and gas were fixed at 2.5 and $01 \mathrm{GPa}$, respectively. The compressional modulus of the mineral phase $\left(M_{S}\right)$ was fixed at $100 \mathrm{GPa}$. The attenuation coefficient $\alpha$ is plotted versus $S_{W}$ and $M_{D r y}$ in Figure 8, left. It is maximum at low gas saturation and $M_{D r y}$ about $0.7 \mathrm{GPa}$. Attenuation decreases as $M_{D r y}$ increases from $0.7 \mathrm{GPa}$ towards higher values and also as $M_{D r y}$ decreases from $0.7 \mathrm{GPa}$ towards smaller values.

In the second example, we examine stiffer rock with $M_{D r y}$ varying between zero and $20 \mathrm{GPa}$. In this case we do not keep porosity constant but instead change it with changing $M_{D r y}$ according to the critical porosity model:

$$
M_{D r y}=M_{S} \frac{\phi}{\phi_{c}} \Rightarrow \phi=\phi_{c} \frac{M_{D r y}}{M_{S}},
$$


where $\phi_{c}$ is the critical porosity. We select $\phi_{c}=0.4$ and keep all other input same as in the first example. The resulting attenuation coefficient is plotted versus $S_{W}$ and $M_{D r y}$ in Figure 8, right. It rapidly decreases with the increasing stiffness of the rock frame.
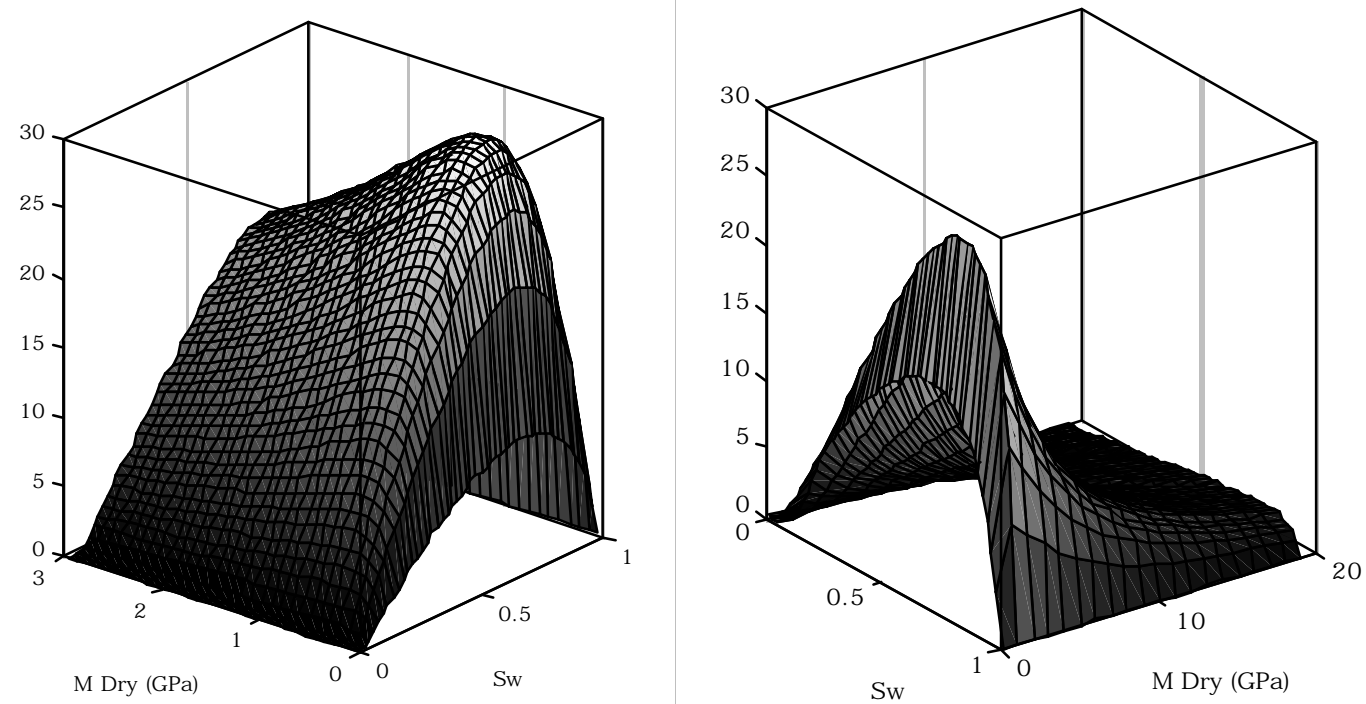

Figure 8. The inverse quality factor versus water saturation and the dry-frame compressional modulus. Left - very soft sand. Right - soft-to-stiff sandstone.

SYNTHETIC EXAMPLES BASED ON WELL LOG DATA

To model attenuation in gas-saturated sediment we select well log data from an interval with alternating sand and shale layers (Figure 9). This is Well 1. The elected interval does not contain hydrocarbons. 

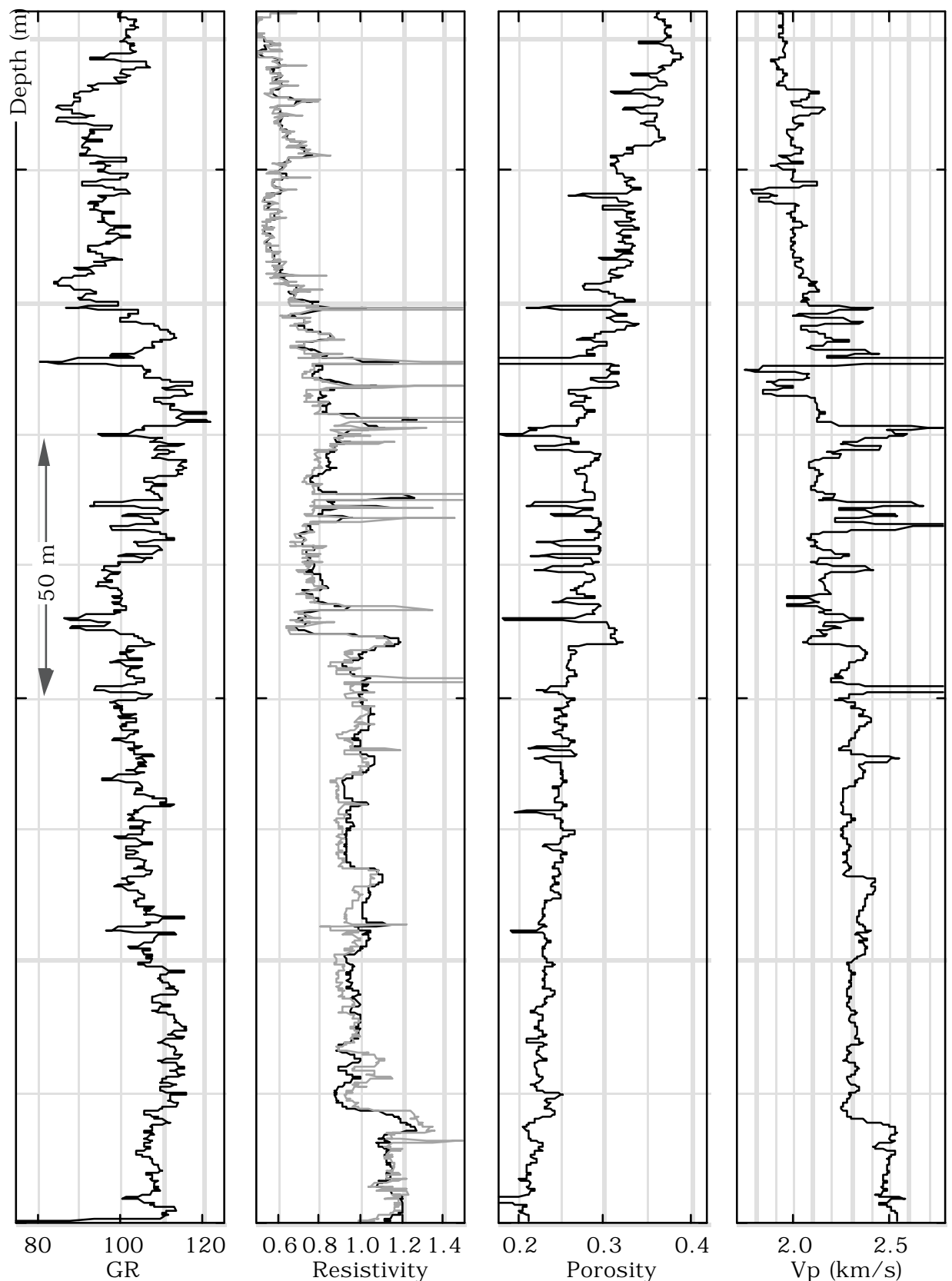

Figure 9. Well 1. Well log data chosen for synthetic attenuation modeling. From left to right - gamma ray; deep (black) and middle (gray) resistivity; total porosity (calculated from bulk density); and the P-wave velocity.

We choose three synthetic scenarios (Figure 10) to introduce gas saturation and calculate the resulting $\mathrm{P}$-wave attenuation. In the first scenario the gas saturation is small, in the second scenario in it in the middle range, and in the third scenario it is large. 

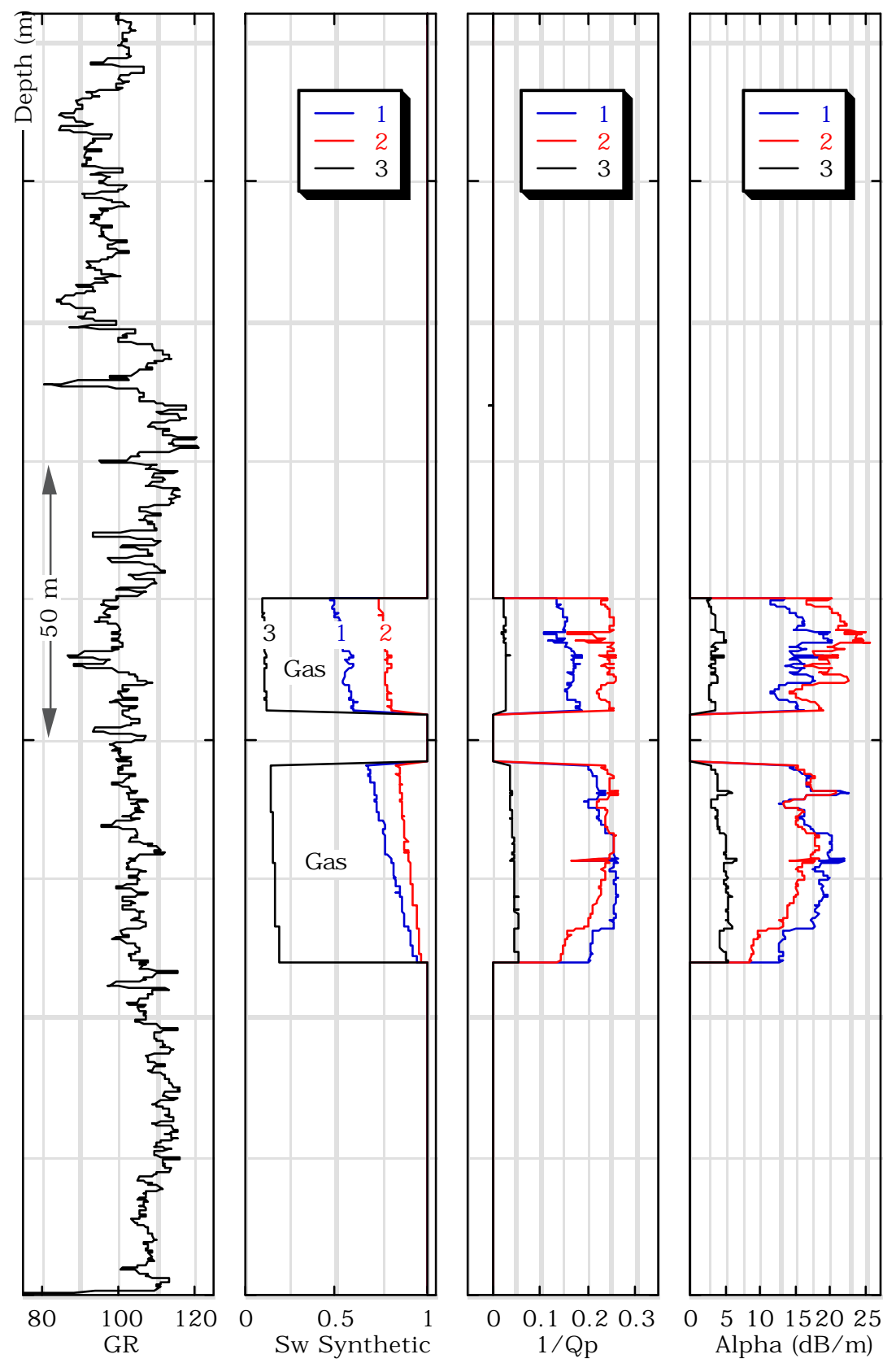

Figure 10. Well 1. Synthetic attenuation modeling for well $\log$ data displayed in Figure 8. From left to right - gamma ray; water saturation for the three synthetic scenarios (color-coded); the inverse P-wave quality factor; and the attenuation coefficient.

In our calculation we used $2.5 \mathrm{GPa}$ for the bulk modulus of water and $0.1 \mathrm{GPa}$ for the bulk modulus of gas. The compressional modulus of the solid phase was $100 \mathrm{GPa}$, constant in the entire interval. In the first two scenarios, the irreducible water saturation was 0.1 while in the third scenario it was 0.01 . 
The results of attenuation calculation are shown as well log curves in Figure 10. The intervals with low and medium gas saturation exhibit attenuation much larger than that in the interval with high gas saturation. High attenuation may be an indicator of noncommercial gas accumulations which otherwise are difficult to discriminate from high gas saturation reservoirs. These results are further emphasized by Figure 11 where the inverse quality factor and attenuation coefficient are plotted versus water saturation for the three synthetic scenarios.
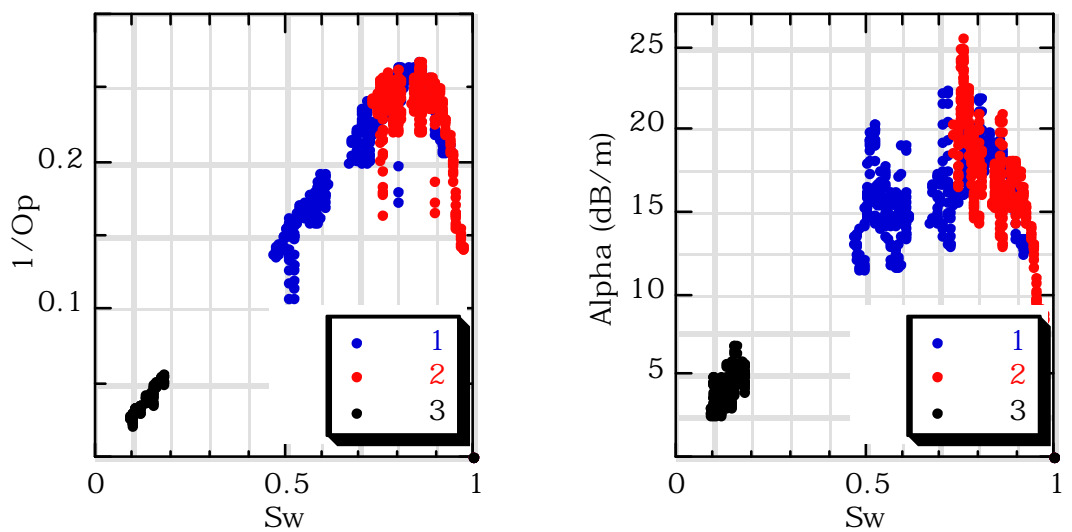

Figure 11. Well 1. Synthetic attenuation modeling for well log data displayed in Figure 8. Left -- the inverse quality factor versus water saturation color coded according to the synthetic scenarios. Right - same for the attenuation coefficient.

The second interval selected for synthetic attenuation modeling is comprised of consolidated low-to-medium porosity sandstone (Figure 12). This is Well 2. The solidphase, water, and gas elastic moduli used are the same as in the previous example. The irreducible water saturation was fixed at 0.01. The calculated inverse quality factor is plotted versus depth in Figure 12. It exhibits the same pattern as in the previous examples - the smaller gas saturation the larger attenuation. Figure 13 where the inverse quality factor and attenuation coefficient are plotted versus saturation further illustrates this effect. 

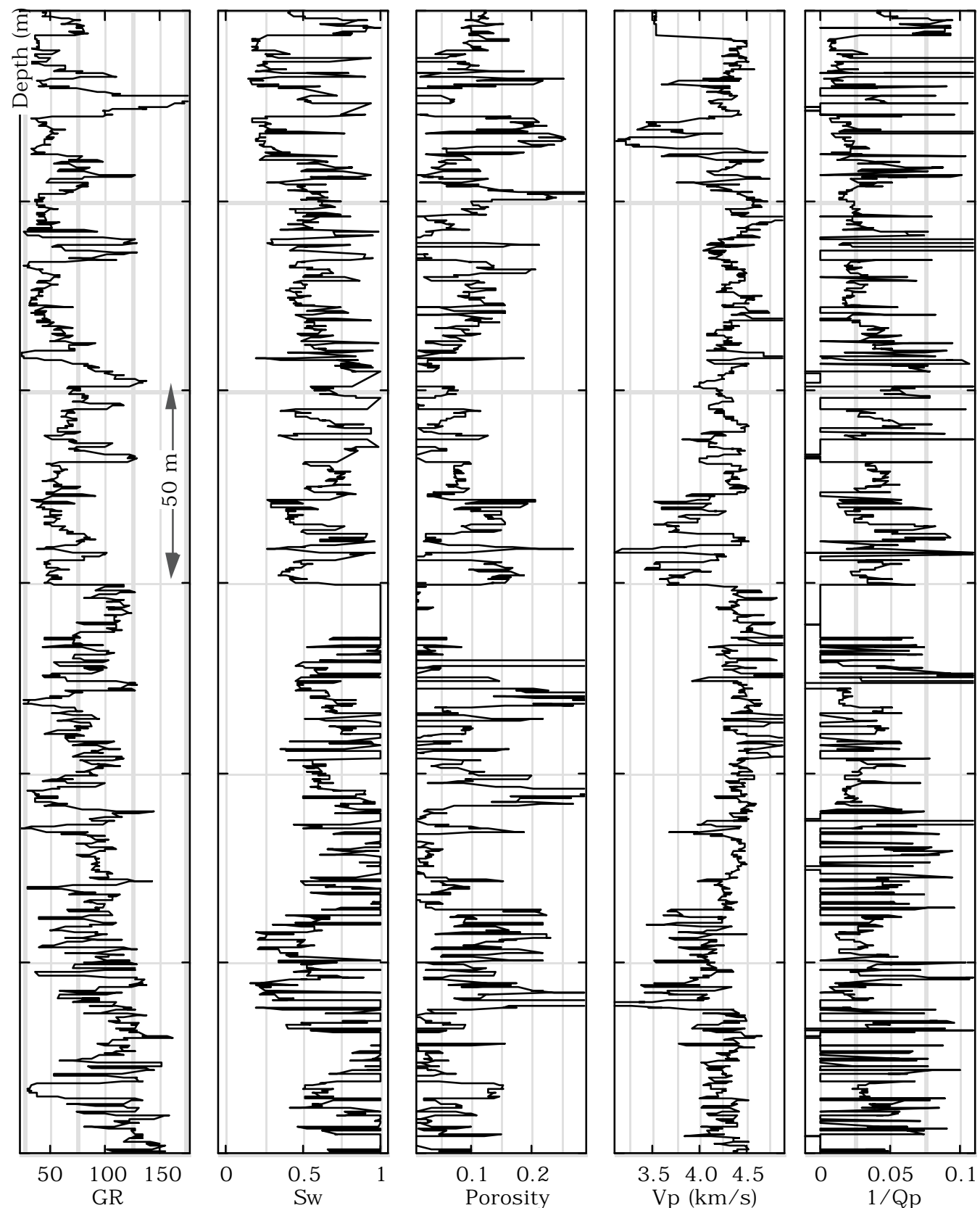

Figure 12. Well 2. Well log curves from a consolidated gas sandstone interval and synthetic inverse quality factor versus depth. From left to right - gamma ray; water saturation; porosity; the P-wave velocity; and the inverse P-wave quality factor.

Let us compare the properties of the two sand intervals examined in the above well log examples. In Figure 14, left, we plot the P-wave impedance versus the total porosity for both intervals (see the well log curves in Figure 10 and 12). The sandstone in Well 2 is of lower porosity and stiffer than in Well 1. This difference is reflected in the inverse 
quality factor versus saturation cross-plot (Figure 14, right). At the same saturation, the attenuation computed in Well 1 is larger than that in Well 2.

This effect directly follows from the fact that the stiffer the rock the smaller the effect of the pore fluid on the bulk modulus. As a result, we observe a reduction in the modulus-frequency dispersion and the accompanying reduction in P-wave attenuation.
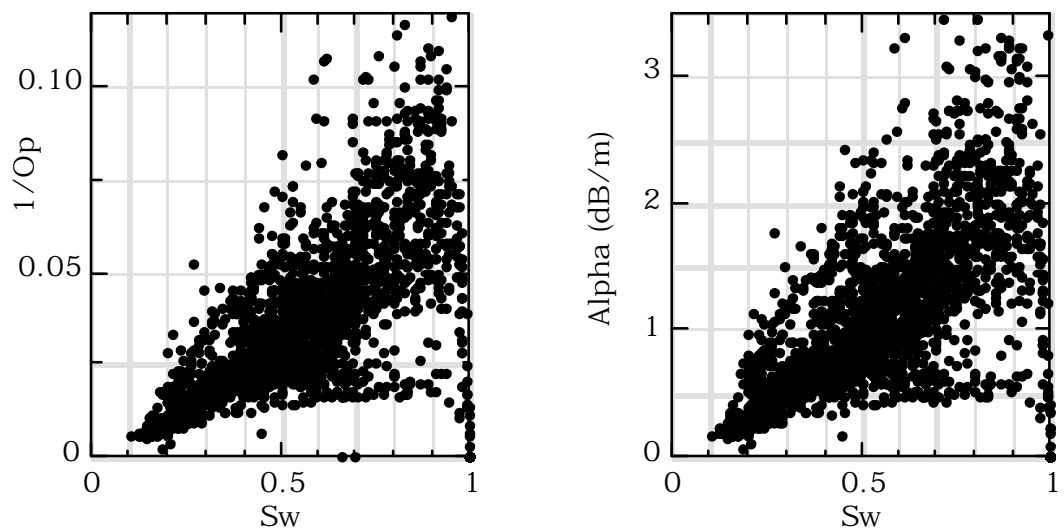

Figure 13. Well 2. Synthetic attenuation modeling for well log data displayed in Figure 11. Left -- the inverse quality factor versus water saturation. Right - same for the attenuation coefficient.
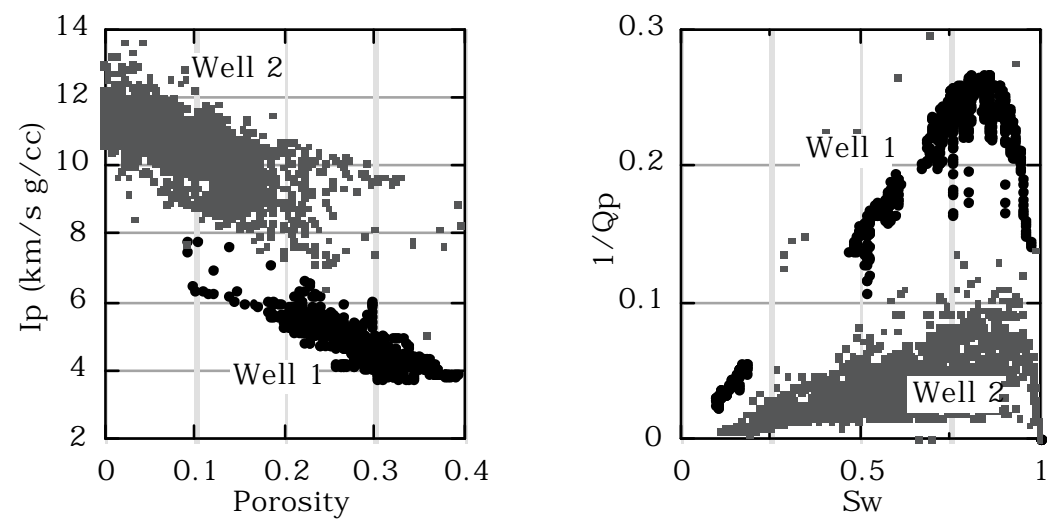

Figure 14. Well 1 and Well 2. Left - the P-wave impedance versus porosity. Right -- synthetic attenuation modeling - the inverse quality factor versus water saturation.

\section{APPLICABILITY OF THE MODEL - DATA AND SPECULATIONS}

There is evidence, albeit extremely sparse, that the patchy saturation pattern does exist and affects the elastic rock properties at well log frequencies in the $1-10 \mathrm{kHz}$ frequency range. A classical example from Cadoret (1993) shows that the saturation pattern (scale) depends on the method of introducing gas into fully saturated rock and that 
this pattern, in turn, affects the velocity in the log frequency range (Figure 15, top). During this experiment, patches of air were visible in a CT-scan images of the rock.

The observed velocity dispersion is matched by $\mathrm{P}$-wave attenuation which peaks at a very low gas saturation (Figure 15, bottom). Cadoret (1993) in fact matched the experimental attenuation data by using the constant $\mathrm{Q}$ attenuation model in combination with the velocity difference (dispersion) between the patchy and homogeneous saturation curves in Figure 15, top. These experimental data confirm the results of the patchy saturation model used to calculate attenuation.
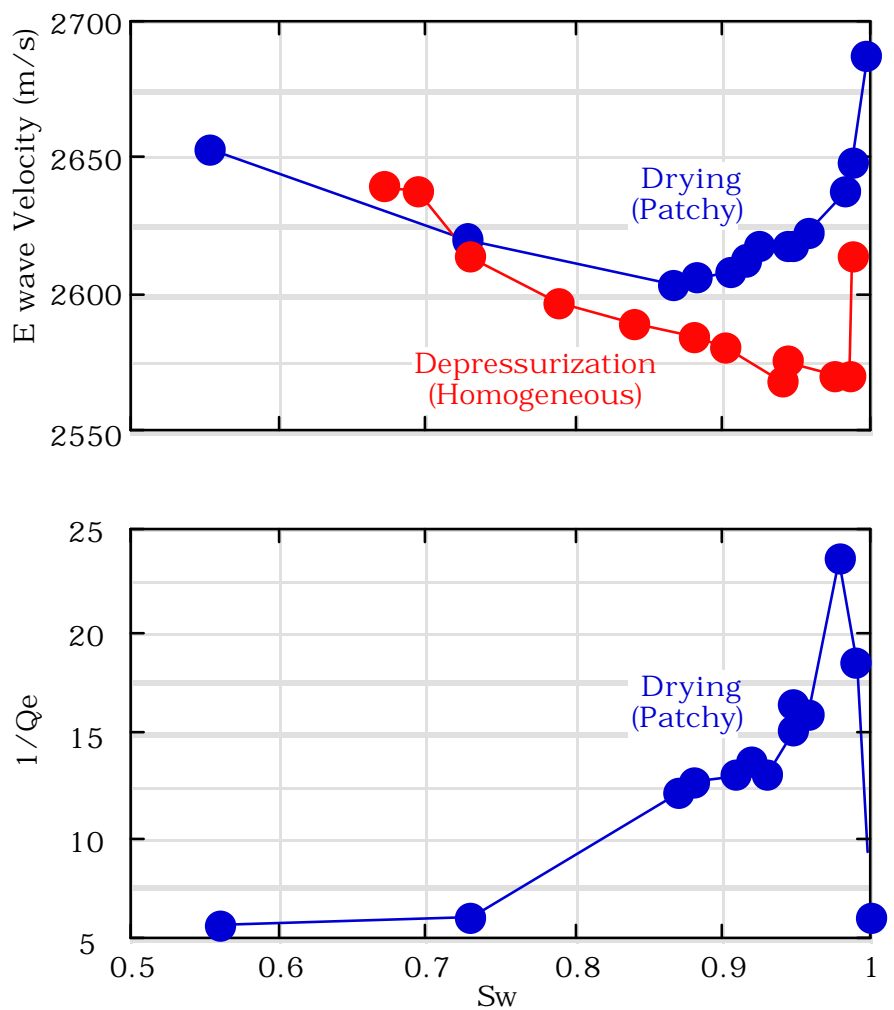

Figure 15. Cadoret's (1993) resonant bar experiment at about $1 \mathrm{kHz}$ frequency. Top - the extensional-wave velocity versus saturation at two saturation patterns resulting from two different methods of introducing gas into the porous saturated rock (marked on the curves). Apparently, the upper curve corresponds to the patchy saturation pattern while the lower curve corresponds to the homogeneous saturation pattern. Bottom - the inverse quality factor apparently resulting from the elastic moduli difference between the homogeneous and patchy saturation patterns.

Murphy (1982) observed the same effect of attenuation peaking at low gas saturation (Figure 16, top), without explicitly identifying patchy saturation. In the same experiment, 
noticeable shear-wave attenuation was observed in partially-saturated rock (Figure 16, bottom). The patchy saturation model introduced in this work predicts that the shearwave attenuation is zero and, therefore, cannot explain the shear-wave attenuation apparent in the Murphy (1982) data.
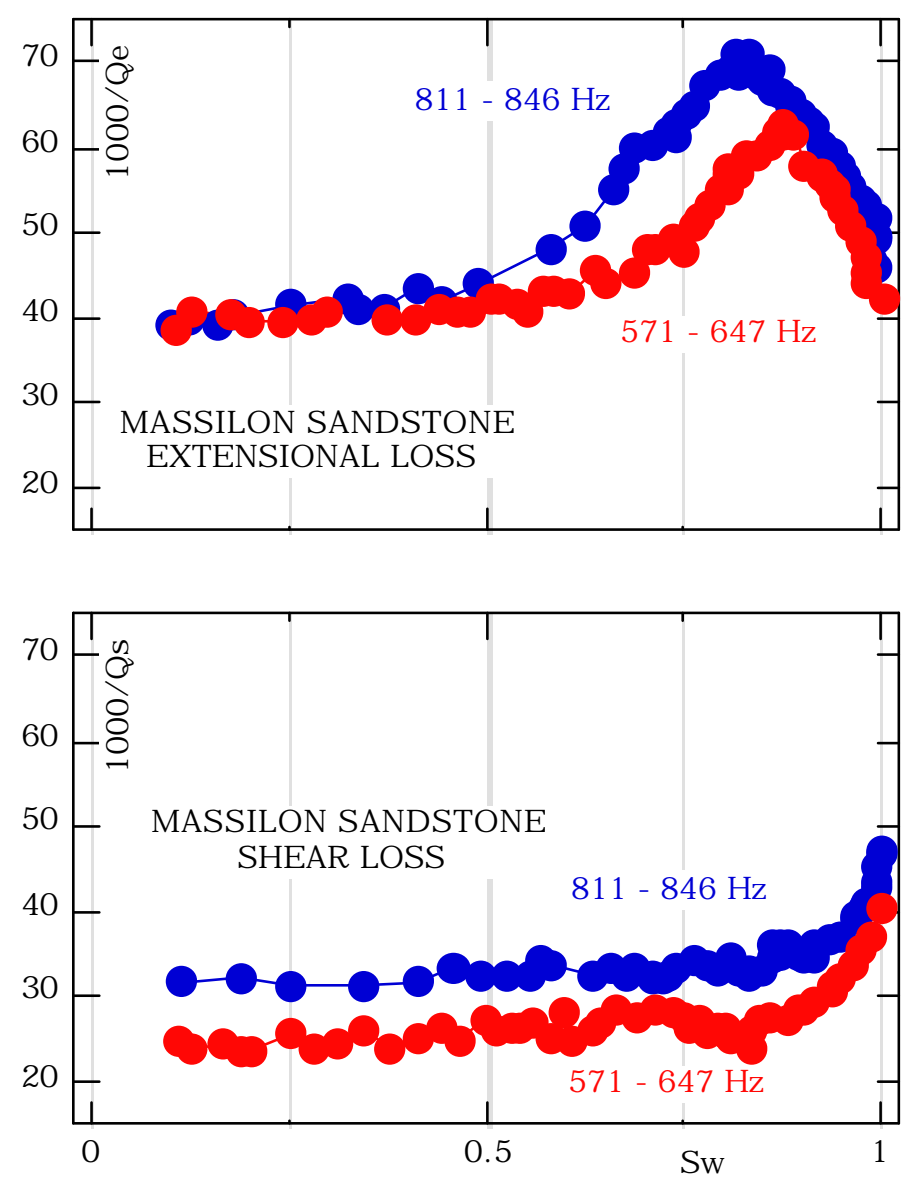

Figure 16. Murphy's (1982) resonant bar experiment in the $0.5-1 \mathrm{kHz}$ frequency range (frequency is indicated on the data curves). The sample is partially saturated Massilon sandstone. Top - the extensional-wave inverse quality factor versus saturation. Bottom -- the shear-wave inverse quality factor versus saturation. The shear-wave attenuation is smaller than the extensional-wave attenuation but is noticeable.

Other experimental data of Murphy (1982) definitely show that the extensional-wave attenuation is related to the presence of liquid in he system (Figure 17). The magnitude of the shear wave attenuation in these experiments is comparable to that of the extensional waves.

How to explain and rationally model the shear-wave attenuation? One way of 
approaching this problem is to use the principle that shear deformation has compression and extension components and that these components do not necessarily cancel each other and still act to generate wave-induced pore fluid flow and the resulting velocity dispersion and attenuation (Mavko and Jizba, 1991). Another possibility is that shear waves reflected at interfaces produce converted P-waves who in turn produce attenuation.

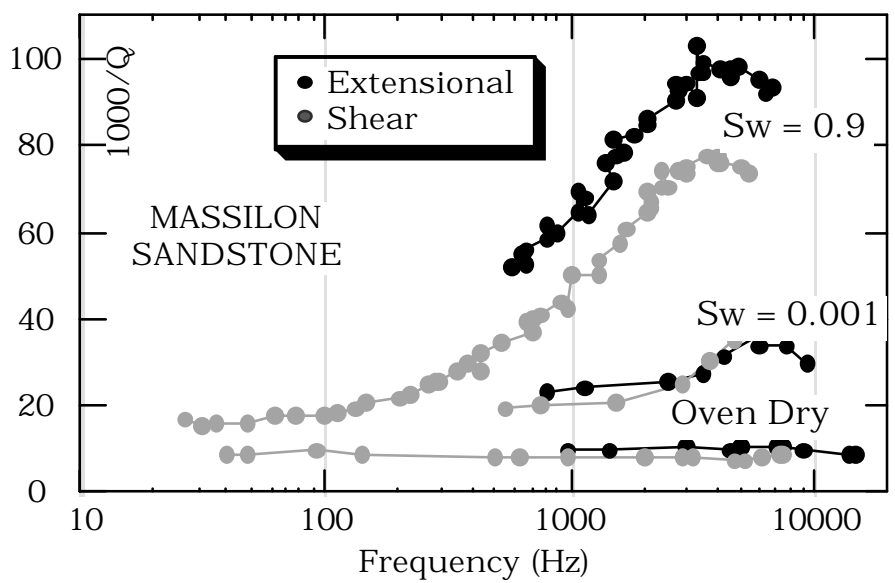

Figure 17. Murphy's (1982) resonant bar experiment on partially saturated Massilon sandstone. The extensional-wave and shear-wave inverse quality factor versus frequency for three saturation values (indicated in the graph). The magnitude of the shear-wave attenuation is comparable to that of the extensionalwave attenuation.

\section{EMPIRICAL MODELS}

Koesoemadinata and McMechan (KOM, 2001) examined laboratory attenuation data available in the literature to statistically relate $Q_{p}$ and $Q_{s}$ to porosity, clay content, water saturation, and frequency.

In Figure 18, left, we plot the inverse quality factor versus water saturation for the synthetic example illustrated in Figure 5. Shown is only one synthetic curve, calculated for $10 \mathrm{MPa}$ effective pressure. In the same frame we plot the P- and S-wave inverse quality factor calculated from KOM using the same input parameters. The KOM $Q_{p}^{-1}$ curve lies below our model curve. So does the $Q_{p}^{-1}$ value calculated from Klimentos and McCann (KLM, 1990). The latter is probably irrelevant to this synthetic example because the original KLM experiments have been conducted on water-saturated consolidated sandstones at $50 \mathrm{MPa}$ effective pressure and $1 \mathrm{MHz}$ frequency and is displayed for reference. 
In Figure 18, right, we plot the inverse quality factor versus water saturation for the synthetic example shown in Figure 6 , for $V_{p}=2.5 \mathrm{~km} / \mathrm{s}$. The appropriate KOM and KLM curves are plotted as well. Neither of the calculated curves matches the values reported by Klimentos (1995).

Let us assume that $\mathrm{KOM}$ regression provides valid estimates for attenuation in fully water saturated rock where our model predicts zero attenuation. Then, by adding the $\mathrm{KOM} S_{W}=100 \% Q_{p}^{-1}$ value to that given by our model for partially saturated curve, we arrive at a synthetic result that approaches the Klimentos (1995) values.

It is also encouraging that $\mathrm{KOM}$ prediction for $Q_{s}^{-1}$ at $S_{W}=100 \%$ matches the Klimentos (1995) values which are about 0.05 at $S_{W}=30 \%$.
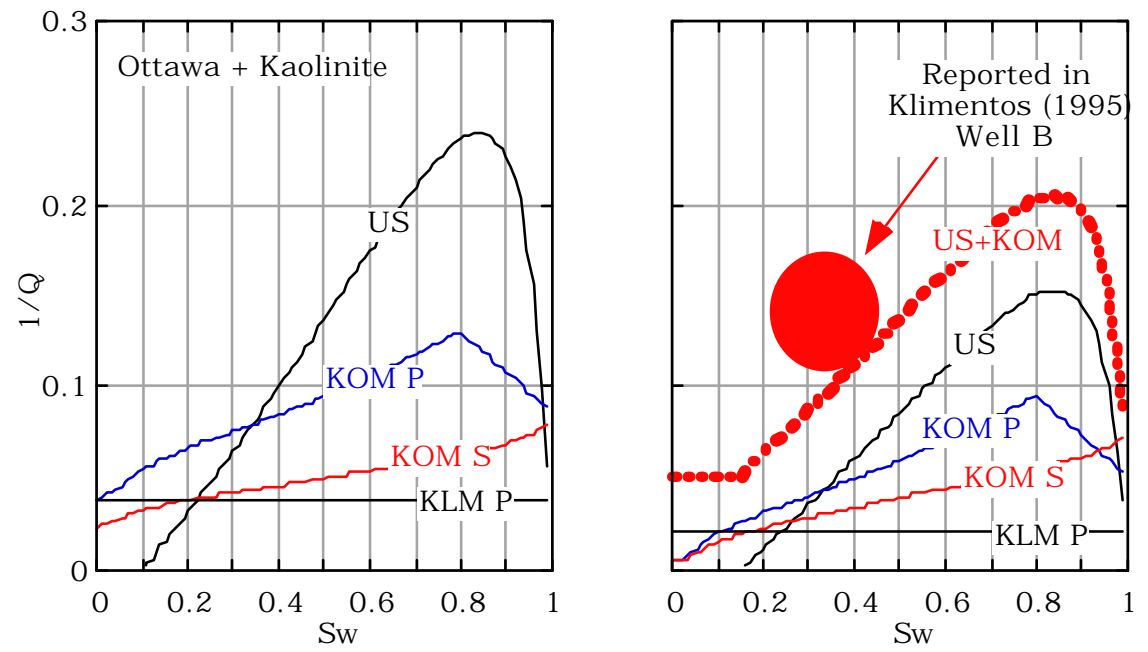

Figure 18. The inverse quality factor versus water saturation using various models. "US" - the patchy saturation model described in this paper; "KOM P" - KOM model for P-wave attenuation; "KOM S"-KOM model for S-wave attenuation; "KLM P" - KLM model for P-wave attenuation; "US+KOM"-combination of the patchy and KOM model where the latter is only used for $100 \%$ water saturation. Left-example illustrated by Figure 5. Right - example illustrated by Figure 6. The large red circle shows values reported by Klimentos (1995).

One conclusion and recipe for practical attenuation prediction that follow from the results shown in Figure 18, right, is to use $\mathrm{KOM} S_{W}=100 \%$ values for "background" $Q_{p}^{-1}$ and $Q_{s}^{-1}$ calculation and add to them values given by our physics-based model. 


\section{COMBINING KOM AND PATCHY MODEL}

We use the method of calculating the background $S_{W}=100 \%$ attenuation from KOM model and then adding attenuation from the patchy saturation model to produce synthetic attenuation values at $S_{W}<100 \%$.

Consider first well log data shown in Figure 9 and 10. The clay content needed for KOM modeling was calculated from GR by linear interpolation and is shown in Figure 19, left frame. The resulting inverse quality factor for $\mathrm{P}-$ and $\mathrm{S}$-waves are plotted versus depth in the same figure for $5000 \mathrm{~Hz}$ frequency. With the S-wave attenuation essentially flat, the P-to-S attenuation ratio will be indicative of gas saturation. 


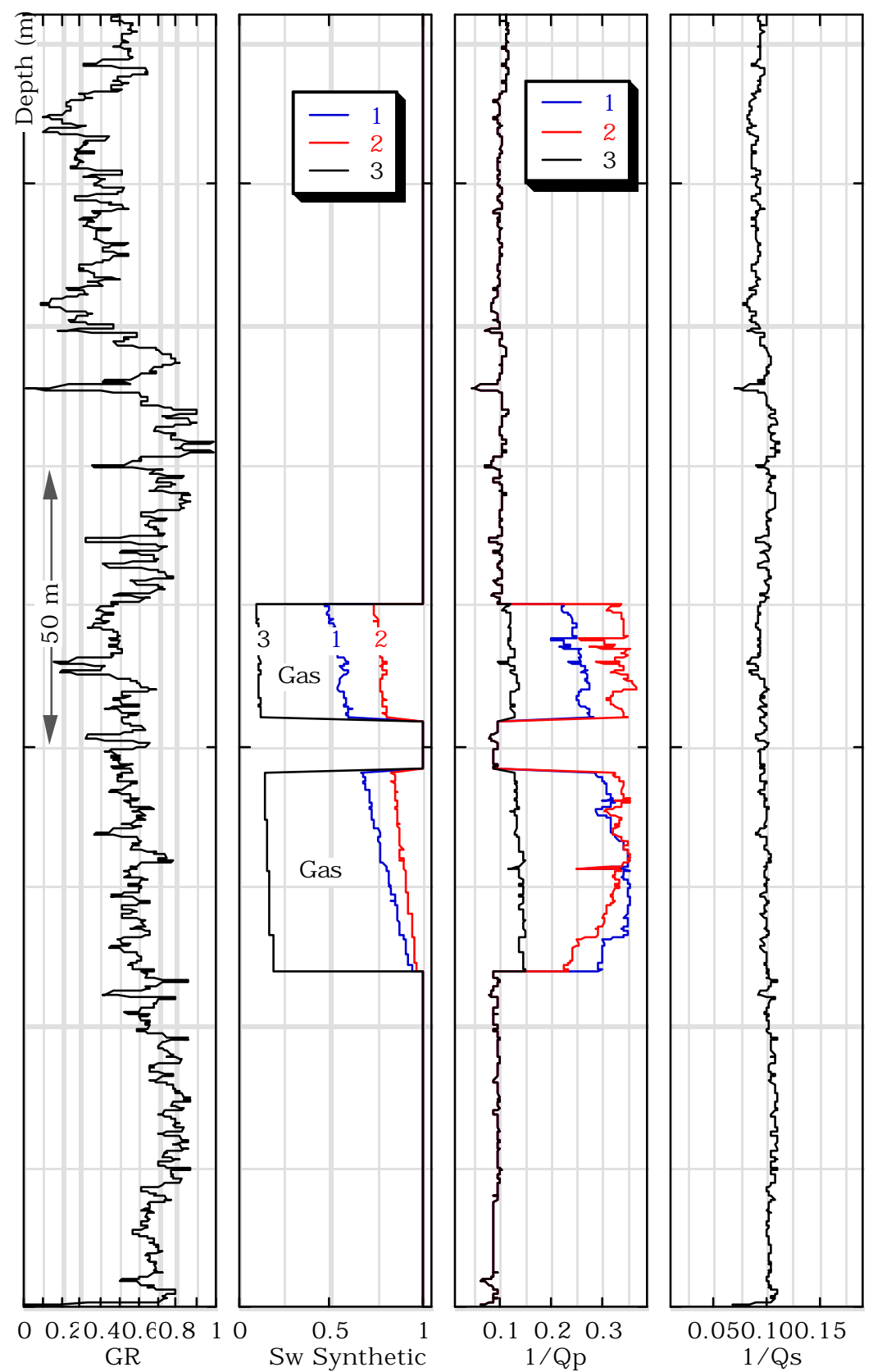

Figure 19. Clay content; water saturation; the inverse P-wave quality factor; and the inverse S-wave quality factor versus depth for the synthetic example shown in Figure 9 and 10 for three saturation scenarios. KOM for $100 \%$ water saturation and the patchy model for varying saturation are simultaneously applied to the same log data.

\section{SANDWICH MODELS FOR SYNTHETIC SEISMIC MODELING}

To produce 3-layer "sandwich" models for synthetic seismic modeling, we use two effective-medium models that predict the effective elastic properties of the dry frame versus porosity and the elastic properties of the mineral phase. The first model is the 
uncemented sand model that uses the modified lower Hashin-Shtrikman curve that connects two end members in the elastic modulus versus porosity plane. The highporosity end member elastic moduli at the critical porosity are calculated from the HertzMindlin contact theory. The zero-porosity end member elastic moduli are those of the mineral phase, calculated from the individual-component elastic moduli via Hill's average.

The second model is for consolidated sandstone. It is the same as the soft-sand model except that instead of the lower Hashin-Shtrikman curve it uses the upper HashinShtrikman curve. Both models accurately mimic the well log data displayed in Figure 9 for a soft shaley sand interval and in Figure 12 for a consolidated sandstone interval (Figure 20).
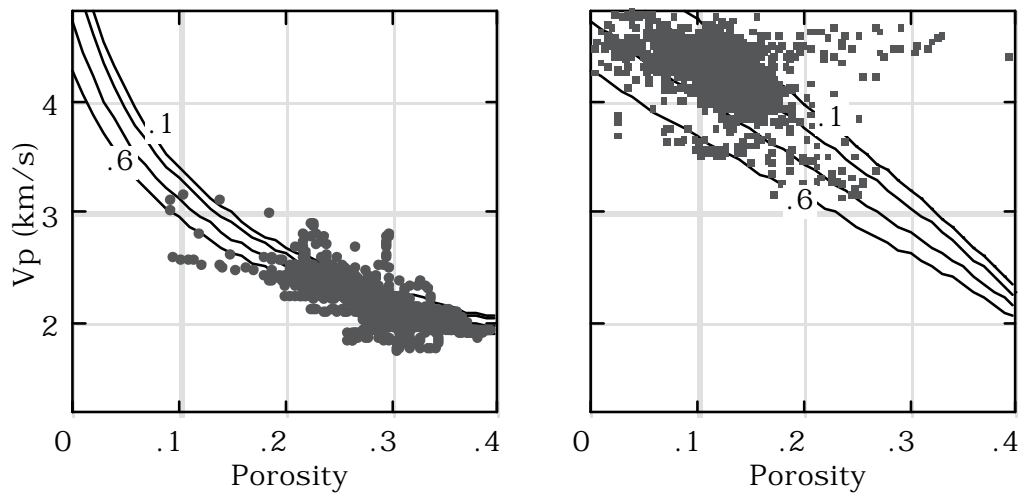

Figure 20. The P-wave velocity versus total porosity. Left - the soft sand interval shown in Figure 9. The symbols are well log data. The curves are from the uncemented sand model, drawn (top to bottom) for clay content $0.1 ; 0.2 ; 0.4$; and 0.6. Clay content is marked for the top and bottom curves. Right - same for the consolidated sandstone interval shown in Figure 12. In both cases the clay content (GR) in the data increase from top bottom and matches the clay content increase in the model curves.

First we generate a soft-sand 3-layer model. We assume that the top shale layer contains $70 \%$ clay and has $35 \%$ total porosity. The bottom shale layer contains $50 \%$ clay and has $30 \%$ porosity. The sand layer contains $5 \%$ clay and has $35 \%$ porosity. The sand contains water and gas. The bulk moduli of water and gas are 2.5 and $0.03 \mathrm{GPa}$, respectively, with the corresponding density of 1 and $0.116 \mathrm{~g} / \mathrm{cm}^{3}$. Within this stratigraphic framework, we consider three saturation scenarios: 100\% water saturation (no gas); $80 \%$ water saturation ("fizz" water); and 20\% water saturation (commercial 
gas). The effective pressure is $20 \mathrm{MPa}$. The corresponding velocity, density, and attenuation values are displayed in Figure 21.

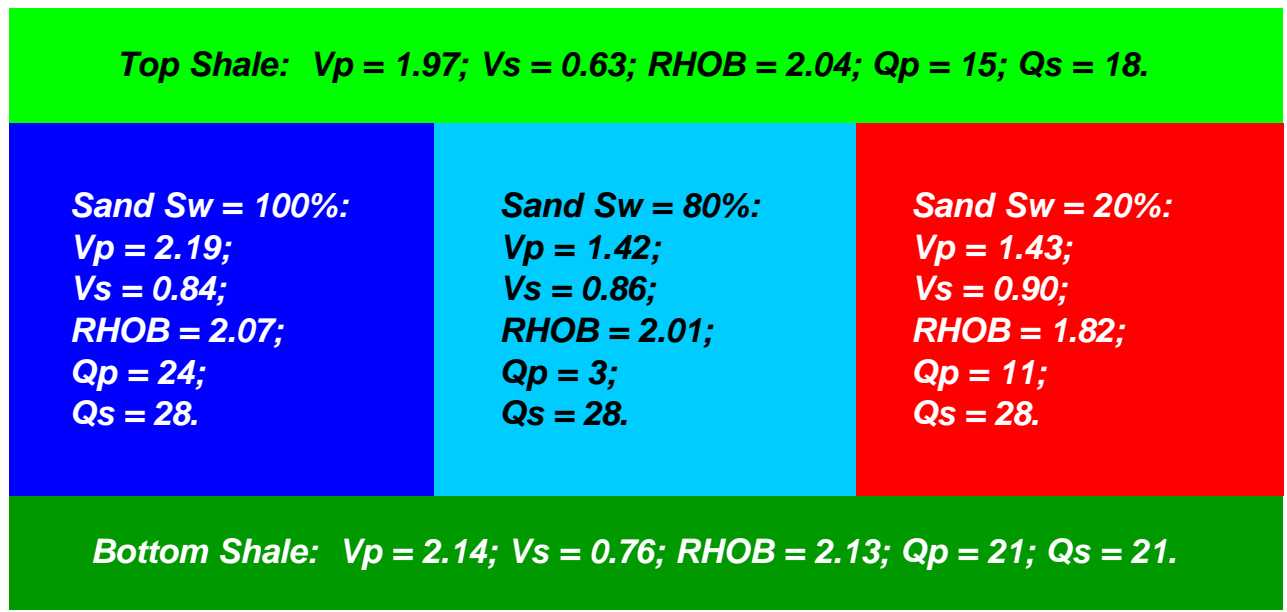

Figure 21. Three soft-sand sandwich models. The top and bottom shale layers are the same in all three cases. The sand's dry-frame properties are also the same. The only variable between the three sand models is water saturation. The velocity is in $\mathrm{km} / \mathrm{s}$

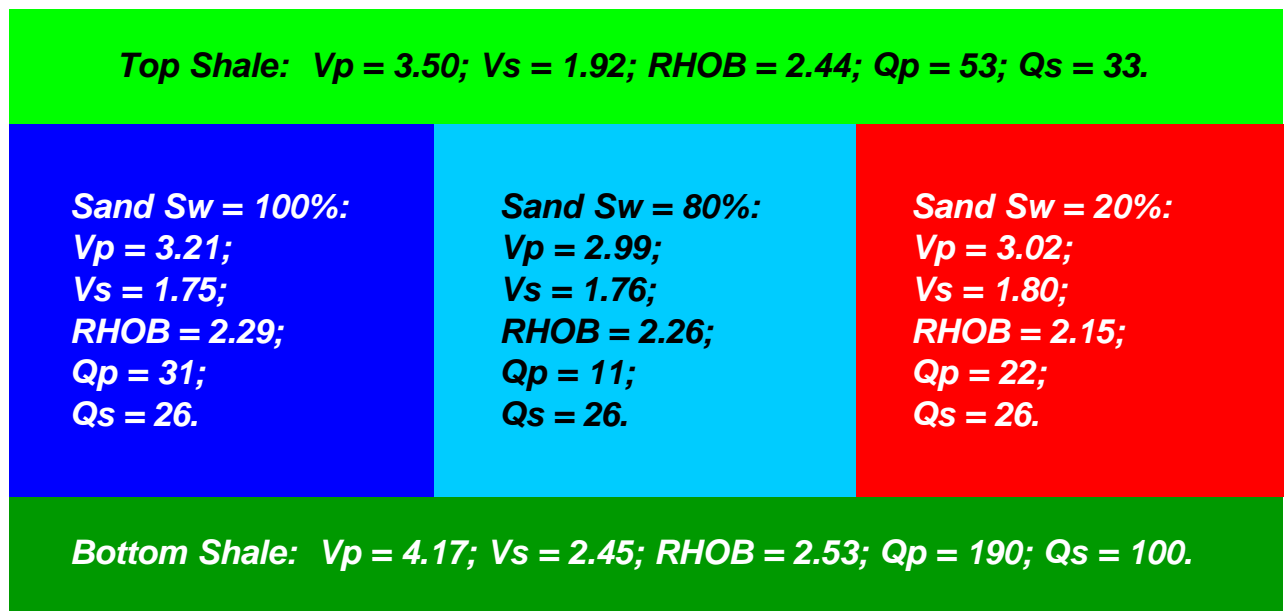

Figure 22. Three consolidated-sand sandwich models. Notations are the same as in Figure 21.

Next we generate a consolidated-sand 3-layer model. We assume that the top shale layer contains $70 \%$ clay and has $10 \%$ total porosity. The bottom shale layer contains $50 \%$ clay and has $5 \%$ porosity. The sand layer contains $5 \%$ clay and has $20 \%$ porosity. The sand contains water and gas. The bulk moduli of water and gas are 2.5 and $0.1 \mathrm{GPa}$, respectively, with the corresponding density of 1 and $0.15 \mathrm{~g} / \mathrm{cm}^{3}$. The saturation scenarios are the same as in the soft-sand model. The effective pressure is $40 \mathrm{MPa}$. The 
corresponding velocity, density, and attenuation values are displayed in Figure 22.

The P-wave attenuation is largest in the "fizz-water" layers. It may be used to seismically discriminate non-commercial gas deposits from commercial intervals.

\section{REFERENCES}

Brie, A., Pampuri, F., Marsala, A.F., and Meazza, O., 1995, Shear sonic interpretation in gas-bearing sands: SPE 30595, 701-710.

Chatenever, A., and Calhoun, J.C., 1952, Visual examinations of fluid behavior in porous media -- Part 1, AIME Petroleum Transactions, 195, 149-195.

Cadoret, T., 1993, Effet de la saturation eau/gaz sur les propriétés acoustiques des roches, Ph.D. thesis, University of Paris.

Dvorkin, J., Moos, D., Packwood, J.L., and Nur, A.M., 1999, Identifying patchy saturation from well logs, Geophysics, 64, 1756-1759.

Klimentos, T., and McCann, C., 1990, Relationships among compressional wave attenuation, porosity, clay content, and permeability in sandstones, Geophysics, 55, 998-1014.

Klimentos, T., 1995, Attenuation of P- and S-waves as a method of distinguishing gas and condensate from oil and water, Geophysics, 60, 447-458.

Knight, R., Dvorkin, J., and Nur, A., 1998, Seismic Signatures of Partial Saturation, Geophysics, 63, 132-138.

Mavko, G., and Jizba, D., 1991, Estimating grain-scale fluid effects on velocity dispersion in rocks, Geophysics, 56,1940-1949.

Mavko, G., Chan, C., and Mukerji, T., 1995, Fluid substitution: Estimating changes in Vp without knowing Vs, Geophysics, 60, 1750-1755.

Murphy, W.F., 1982, Effects of microstructure and pore fluids on the acoustic properties of granular sedimentary materials, Ph.D. thesis, Stanford University.

Yin, H., 1993, Acoustic velocity and attenuation of rocks: Isotropy, intrinsic anisotropy, and stress-induced anisotropy, Ph.D. thesis, Stanford University. 


\section{SECTION 2: Q, ABSORPTION AND DISPERSION COMPUTATION BY SEVERAL DIFFERENT METHODS}

\section{INTRODUCTION:}

This paper is written to describe the computational details of the "Q-AttenuationDispersion" computation "QADCOM" program, which is now under development. The program is written as a FOCUS module. Input will be NMO applied CDP or image gathers, or stacked or migrated sections. Users will input reflection times at several locations along the given seismic lines. QAD will be computed between given events. More than two events may be input. The program will compute QAD with several different methods and output results in trace and printed form.

\section{COMPUTATION METHODS:}

We will utilize several published and experimental methods to compute QAD. In this paper, we will describe the computational methods, as we have coded them in Fortran. Fortran subroutines will be written separately for each method to allow the members to be able to select individual routines for their own use.

\section{I: SPECTRAL DIVISION:}

This method uses the amplitude spectra computed for events representing the top and bottom of the analyzed beds and forms the log amplitude ratio to determine the $\mathrm{Q}$ factor. Absorption and dispersion are computed from the log amplitude spectral differences. The constant $\mathrm{C}$ accommodates the reflectivity differences between the events.

$$
\left|A_{t}(f)\right|=\left|A_{0}(f)\right| \cdot \exp (-\pi f t / Q)+C
$$

In this program, we will use Gabor-Morlet Joint Time Frequency decomposition to compute the instantaneous spectrum of the traces (Morlet et al, 1982, Koehler, 1983, Taner, 2001). The decomposition will allow us to have controlled frequency and time domain resolution. We will consider both the cases of frequency independent as well as frequency dependent QAD.

\section{II: THE RISE TIME METHOD:}

A sharper rise time in the onset of a seismic wavelet represents wider bandwidth, thus low absorption. Therefore the rise time could be used to detect anomalous absorption zones. In previous reports the rise time was measured from the seismic traces, which gives different results depending on the phase of the seismic wavelet. We will now use the rise time measured in the envelope of the complex wavelet. Since the envelope is not affected by the phase characteristics, the method will not depend on the phase of the wavelet. The relation of rise time to $\mathrm{Q}$ is given by; 
$\tau=\tau_{0}+c .\left(t-t_{0}\right) / Q$

(2)

Here $\tau$ is the rise time, namely, the maximum envelope amplitude divided by the maximum slope of the leading edge of the wavelet. $\boldsymbol{\tau}_{0}$ is the rise time of a reference wavelet, the constant $\mathrm{c}$ is generally dependent on the magnitude of $\mathrm{Q}$, and usually lies between 0.485 and 0.1 . " $\mathrm{t}$ " represents the travel time. Since the computation is performed between the events representing the top and the base of a particular bed, the rise time of reference wavelet is measured from the top event and the travel time is the time difference between the two events.

Here we assume that we are measuring the rise times at the top and the base of a bed of a propagating wave front. This implies that we are not affected by complicated reflectivity responses at either end of the bed. Hence, this method can be used in lab experiments. Unfortunately, in real seismic data cases we will have variable reflectivity conditions. In the simplest case the reflection coefficient will vary, affecting the event amplitudes. Therefore, we have to modify the rise time to be dependent of event magnitude. We propose to use inverse of the maximum slope of the onset of the events. This will still have the rise time information, except $t$ is with respect to unit envelope height.

\section{III: FREQUENCY SHIFT METHOD:}

Complex trace analysis provides a number of fundamental parameters of the propagating wave front. It can be shown that the instantaneous frequency computed at the time corresponding to the maxima of the envelope is the average frequency of the Fourier amplitude spectrum of the corresponding wavelet (Barnes, 1991). If we assume that the wavelet has a reasonably uncomplicated spectral shape (trapezoidal, Butterworth, Ricker or Gaussian shaped amplitude spectrum), then a shift of the mean of the spectrum will indicate absorption. Therefore, based on the assumed shape of the wavelet, we can relate the average frequency shift to the absorption coefficient $\alpha(\omega)$ or to the $\mathrm{Q}$ factor.

Let $f(t)$ represent the recorded seismic data and let $g(t)$ be its Hilbert transform., the complex trace is given by (Taner et al 1979);

$$
\tilde{f}(t)=f(t)+i \cdot g(t)
$$

This can be expressed in polar form as;

$$
\tilde{f}(t)=e(t) \exp (i . \varphi(t))
$$

where $\mathrm{e}(\mathrm{t})$ is the instantaneous envelope (modulus of the complex trace) and $\boldsymbol{\varphi}(\boldsymbol{t})$ is the instantaneous phase (argument of the complex trace). They are related to the real and imaginary parts as;

$$
\begin{aligned}
& e(t)=\sqrt{f^{2}(t)+g^{2}(t)} \\
& \varphi(t)=\arctan \{g(t) / f(t)\}
\end{aligned}
$$


The instantaneous frequency is the time rate of change of the instantaneous phase. The point in time at which the envelope is at its maximum is the point in time at which all the frequency components of the propagating wave are in phase. The maximum develops by the in-phase addition of all of the frequencies. Therefore instantaneous phase and its derivative have statistical significance. Here, we will follow Barnes' (1991) procedure to show how the instantaneous frequency relates to the Fourier transform. Assume that we are computing the Fourier transforms at time $t=0$. Let the frequency domain representation of the complex trace be given as;

$F(\omega)=A(\omega) \cdot \exp \{i \phi(\omega)\}$

Then its inverse transform back to time domain is given by;

$\tilde{f}(t)=\frac{1}{2 \pi} \int_{0}^{+\infty} A(\omega) \cdot \exp \{i \phi(\omega)\} \cdot \exp (i \omega t) \cdot d \omega$

If we compute the derivative of the complex trace and divide it by this complex trace, (we do this in frequency domain) the time and frequency domain relationship will be ;

$$
\frac{\tilde{f}^{\prime}(t)}{\tilde{f}(t)}=\frac{i \int_{0}^{\infty} \omega A(\omega) \exp \{i \phi(\omega)\} \exp (i \omega t) d \omega}{\int A(\omega) \exp \{i \phi(\omega)\} \exp (i \omega t) d \omega}
$$

This equation turns out to be the first moment representation of the complex trace amplitude spectrum. Therefore it determines the centroid frequency. Equation 8 can be written as (referring to equation 4);

$$
\frac{\tilde{f}^{\prime}(t)}{\tilde{f}(t)}=\frac{d}{d t} \ln \{\tilde{f}(t)\}=\frac{d}{d t}\{\ln [a(t)]\}+\frac{d}{d t}\{i \varphi(t)\}
$$

The first term indicates the time rate of change of envelope, which is zero at the envelope peak. The second term is the time rate of change of the phase angle, or the instantaneous frequency. Equation 8 indicates that this frequency is the centroid of the amplitude spectrum.

Let us now consider a wavelet with a Gaussian shaped amplitude spectrum;

$$
W(\boldsymbol{\omega})=W_{0} \exp \left\{-\beta\left(\boldsymbol{\omega}-\boldsymbol{\omega}_{0}\right)^{2}\right\}
$$

where $\boldsymbol{\omega}_{0}$ is the spectrum's center frequency, and $\beta$ is an arbitrary shape factor, relating to the bandwidth. The log amplitude scale representation of this wavelet is then;

$\ln W(\omega)=\ln W_{0}-\beta\left(\omega-\omega_{0}\right)^{2}$

If the wavelet travels through an absorbing medium, its spectrum will undergo modification. Since the distance absorption coefficient $\alpha$ is

$$
\alpha=\omega / 2 v Q
$$


and the temporal absorption coefficient

$$
a=v \alpha=\omega / 2 Q
$$

Then the modified spectrum will be in the form;

$\ln W_{1}(\omega)=\ln W_{0}-\beta\left(\omega-\omega_{0}\right)^{2}-\omega t / 2 Q$

We can determine the new spectral maximum of the now attenuated wavelet by computing the derivative of equation 12 and setting it equal to zero;

$$
\frac{d}{d \omega} \ln W(\omega)=-2 \beta\left(\omega_{c}-\omega_{0}\right)-t / 2 Q=0
$$

We finally solve for the new centroid frequency $\boldsymbol{\omega}_{c}$, namely;

$\omega_{c}=\omega_{0}-t / 4 Q \beta$

Since all variables in Eq.14 are positive, the new centroid $\boldsymbol{\omega}_{c}$ will be lower than before; this centroid frequency lowering is a function of the travel time and of $Q^{-1}$.

If the seismic processing is performed with a controlled wavelet shape, then a specific relationship between frequency shift and $\mathrm{Q}$ can be developed. For arbitrarily shaped wavelets, this method, obviously, will give approximate results. Note that, here we are assuming a single interface. For complicated cases, the resulting spectrum will be too complicated to provide reliable results. The Gaussian shape factor $\boldsymbol{\beta}$ relates to the instantaneous shape factor given by Eq. 25. Therefore we could use the instantaneous bandwidth attribute as a reasonable estimate of $\boldsymbol{\beta}$.

\section{IV: MINIMUM PHASE INVERSE SYNTHETIC DIVISION METHOD:}

This method will produce long wavelength estimate of QAD values. This is because the minimum phase inverse operators are computed from autocorrelation functions determined over fairly long time windows. To obtain a stable autocorrelation function one needs a time window at least 6 to 10 times the selected maximum lag. If the events are not well separated in time, then their effects will be combined. On the other hand, since all computations are carried by minimum phase wavelets, we satisfy the KramersKronig conditions of causality. The process starts with the computation of autocorrelations taken over windows centered around events of interest. From these we compute minimum phase inverse operators (Taner, 1996). Let $\boldsymbol{W}_{1}^{-1}(z)$ and $\boldsymbol{W}_{2}^{-1}(z)$ be the inverses of the $z$-transforms $W_{1}(z)$ and $W_{2}(z)$, where $W_{1}(z)$ and $W_{2}(z)$ are the $\mathrm{z}=$ transforms of the events reflected from the top and from the bottom of a bed, respectively. Then the operator corresponding to the spectral ratio is determined by a synthetic division ;

$$
R(z)=W_{1}^{-1}(z) / W_{2}^{-1}(z)
$$


The frequency domain representation of $\mathrm{R}(\mathrm{z})$ will contain both the $\mathrm{Q}$-absorption and dispersion effects. Since the result of the division (Eq. 15) is also minimum phase, it satisfies the Kramers-Kronig causality condition. The Frequency domain representation is now;

$\ln \left\{W_{2}(\omega) / W_{1}(\omega)\right\}=\ln |R(\omega)|+i \phi(\omega)$

where the term; (referring to Eq. 1 )

$\ln |R(\omega)|=-\omega \cdot t / 2 Q$

$\phi(\omega)$

represents absorption, while the term

represents dispersion. Note, that the amplitude and phase spectra of a $\mathrm{R}(\mathrm{z})$ indicate respectively how both absorption $\mathrm{Q}$ and dispersion all depend on frequency $\boldsymbol{\omega}$.

\section{V： SECOND METHOD FOR SYNTHETIC DIVISION: ADAPTIVE COMPUTATION:}

In this method, we will compute minimum phase inverse operators in an adaptive and continuous manner. The synthetic division requires a time window 6 to 10 times the autocorrelation and operator length. This will give us long wavelength estimates of absorption. In this second method, we will use the adaptive computation of the minimum phase operators that will be more reflective of local changes. First, we will give a short review of adaptive computation of the operators, (Widrow and Stearns, 1985). The prediction error is the difference between the actual and predicted values of the seismic trace (Consider the data is sampled at $\Delta t$ increments);

$\varepsilon=f(t)-\tilde{f}(t)$

where, the predicted trace is computed $\mathrm{b}$ the convolution;

$$
\tilde{f}(t)=\sum_{n=0}^{N} w(n) \cdot f(t-n)
$$

In the conventional computation, we compute the operator $w(n)$ that minimizes the sum of squares of the error over a time window;

$$
\varepsilon^{2}=\sum_{t=T_{1}}^{T_{2}}\left\{f(t)-\sum_{n=0}^{N} w(n) . f(t-n)\right\}^{2}=\min .
$$

The desired minimization is achieved by taking the partial derivatives of the error function with respect to the unknown operators $\mathrm{w}(\mathrm{n})$ and setting them equal to zero. The partial derivatives represent the tangent (gradient) of the error surface at the coordinates of $w(n)$. By setting the partial derivatives equal to zero, we compute the coordinates of minimum of the error surface in terms of $w(n)$. We use this concept in the adaptive updating of the operators. 
We start with computation of the operators in the conventional manner over a time window located at the shallow part of the seismic section. This operator will be used as

the seed. The error at each sample is;

$$
\varepsilon^{2}(t)=\left\{f(t)-\sum_{n=0}^{N} w(n) \cdot f(t-n)\right\}^{2}
$$

The local gradient will be ;

$$
\partial \varepsilon^{2}(t) / \partial w(m)=-2 . f(t-m)\left\{f(t)-\sum_{n=0}^{N} w(n) . f(t-n)\right\}
$$

If we are at the minimum of the error surface, all of the partial derivatives will be equal to zero. If we are not at the minimum position, the gradient will point toward an increasing direction of the error surface. To reach the maximum, we will have to adjust the operators in the opposite direction of the gradient. This is the essence of the Steepest Descent method. However, the amount of adjustment is usually a small fraction of the amount of error. This assures the stability of the process. The fraction is called the "Learning Rate". The steepest descent is simply the negative of the derivative shown in equation 22 . The update of the operators will be in the steepest descent direction;;

$$
w^{t+1}(m)=w^{t}(m)-\eta \cdot \frac{\partial\left[\varepsilon^{2}(t)\right]}{\partial w(m)},
$$

Therefore the adaptive adjustment of the operators at each sample will be;

$$
w^{t+1}(m)=w^{t}(m)+\eta \cdot f(t-m)\left\{f(t)-\sum_{n=0}^{N} w^{t}(n) \cdot f(t-n)\right\}
$$

where, superscript $t$ represent the set of operator weights at time sample $t$, and $\boldsymbol{\eta}$ is the learning rate. The learning rate will be on the order of 0.01 . In conventional processing the amount within the bracket in equation 23 actually represents the deconvolved output. Therefore, we can simplify the expression to;

$$
w^{t+1}(m)=w^{t}(m)+\eta \cdot f(t-m) \varepsilon(t)
$$

Widrow and Stearns (1985) have shown that this recursion is stable and that it continually adapts itself toward the optimum solution. The operators corresponding time $t$ are updated by a small amount proportional to the error multiplied by the m'th data sample. The updated operators are then used to predict the next data sample at time $t+1$. The recursion given by equation 24 provides us with an operator for each data sample, which varies continuously along the seismic trace. We save a subset of these operators, computed around some significant geological boundaries and perform the synthetic division as described in the first method.

\section{VI: RELATIVE Q FROM COMPLEX TRACE ATTRIBUTES:}

Barnes (1993) defines "instantaneous bandwidth" as the ratio of the time rate of change of the envelope to envelope magnitude; 
$\sigma_{i}^{2}(t)=\left[\frac{d a(t) / d t}{2 \pi a(t)}\right]^{2}$

$\mathrm{He}$ also calls this the 'instantaneous decay rate'. This rate is closely related to the rise time (see Section II). Since faster rise times will be associated with lesser decay rates, hence with wider bandwidth. This bandwidth estimate can be used as the Gaussian shape factor $\boldsymbol{\beta}$ in the frequency shift method discussed in Section III above. Barnes, defines an instantaneous $\mathrm{Q}$ factor as;

$$
Q_{i}(t)=-\frac{\pi f_{i}(t)}{\sigma_{i}(t)}
$$

where the subscript " $\mathrm{i}$ " represents an instantaneous attribute and where $f(t)$ is an instantaneous frequency attribute. The minus sign indicates amplitude decay. In the computation we substitute equation 25 into absolute form of equation 26. This simplifies instantaneous Q to;

$$
Q_{i}(t)=\frac{2 \pi^{2} f_{i}(t) \cdot a(t)}{|d a(t) / d t|}
$$

It is obvious that this expression will give different results for wavelet onset times than for wavelet back side times. This is related to the rise time computation. Hence, meaningful results may be obtained if a(t) is determined at the peak of the envelope, and the time derivative is computed at the onset of the reflections where the envelope slope is maximum.

\section{VII: RELATIVE Q FROM COMPLEX TRACE ATTRIBUTES - SECOND DERIVATIVE OF ENVELOPE:}

This method is similar to the rise time method. The second derivative computed at the maxima of the trace envelopes measures the curvature. The curvature relates to the bandwidth and the frequency content of the events. If the curvature is large, then the envelope represents an event with wider bandwidth, containing higher frequencies. If the events have been attenuated, the curvature will become softer, that is, the second derivative will become smaller. Since the second derivative is computed at he maxima of the trace envelope, it is a wavelet attribute, hence it is related to the Fourier transform of the event.

Computationally, the second derivative is approximated by the second difference of sampled data. It will be affected by the event magnitude. Hence we will compute the second difference normalized by the envelope magnitude at the maxima. The relative estimate of $\mathrm{Q}$ is given by;

$$
\tilde{Q}(t)=\frac{\partial^{2} e(t) / \partial t^{2}}{|e(t)|_{(\max )}}
$$


We now show that the second derivative of the envelope is a relative indicator of absorption and i.e., of Q. If we assume the envelope of the wavelet is a Gaussian with shape factor $b$,

$$
e(t)=A \cdot \exp \left(-b t^{2}\right)
$$

Then, its derivatives will be;

$$
\begin{gathered}
d e(t) / d t=-2 A \cdot b t \cdot \exp \left(-b t^{2}\right) \\
d^{2} e(t) / d t^{2}=A \cdot\left(4 b^{2} t^{2}-2 b\right) \exp \left(-b t^{2}\right)
\end{gathered}
$$

The second derivative computed at $\mathrm{t}=0$ will be;

$$
\left[d^{2} e(t) / d t^{2}\right]_{t=0}=-2 A \cdot b
$$

The second derivative of the Gaussian shaped envelope is equal to twice the shape factor times the amplitude at its maximum. As we will see from equations 43 and 44 (below) the shape factor relates to the frequency bandwidth of the wavelet under the envelope;

$$
\Delta \omega_{0}=4 \sqrt{b \cdot \ln 2}
$$

Therefore, a decreasing second derivative of the envelope will indicate a corresponding reduction of the bandwidth. The instantaneous frequency measured at the peak of the envelope will give the location of the centroid of the frequency band. Waves propagating through a dispersive medium will experience central frequency shifts to lower frequencies and bandwidth will become narrower. The second derivative of the envelope gives an additional indication about the spectral change.

Equation 14 above shows the relation between the frequency shift and inverse $\mathrm{Q}$ and the

Gaussian shaped spectrum shaping factor $\beta$. Equation 34 or 44 gives the relationship between time and frequency domain shaping factors. If we assume a Gaussian spectral shape, then we can estimate $\mathrm{Q}$ from the second derivative as we could from the frequency shift. We estimate the time domain shaping factor from equation 31 , by dividing the second derivative of the envelope by the envelope magnitude at the maximum. We substitute equation 43 into equation 14 and get;

$$
\omega_{c}=\omega_{0}-\Delta t . b / Q
$$

Making appropriate substitutions and solving for Q we obtain;

$$
Q=\frac{\Delta t \cdot b}{2 \pi\left(f_{0}-f_{t}\right)}
$$

This expression involves estimating the second derivative of the envelope, rather than the shape of the spectrum in the frequency domain. The frequencies needed for the equation 34 are the wavelet frequencies, also computed at the maximum of the envelope.

\section{VIII: RELATIVE Q FROM WAVELET WIDTH-vs.-BAND WIDTH RELATION:}


This is somewhat similar to the second derivative of the envelope. The method is based on the relationship between the wavelet width in the time domain and the bandwidth in the frequency domain. We will use Gabor wavelets to demonstrate the relationship.

The time domain expression for the Gabor wavelet is:

$g_{j}(t)=\exp \left(-b_{j} t^{2}\right) \cdot \exp \left(i \omega_{j} t\right)$ :

The corresponding frequency domain response is:

$G_{j}(\omega)=\int_{-\infty}^{\infty} g_{j}(t) \exp (i \omega t) d t=\sqrt{\frac{\pi}{b_{j}}} \cdot \exp \left\{-\left(\omega-\omega_{j}\right)^{2} / 4 b_{j}\right\}$

$\Delta t_{j}=k_{1} / \omega_{j}$

width of $j$ 'th wavelet in time domain,

(37)

$\Delta \omega_{j}=k_{2} \omega_{j}$

width of $j$ th wavelet in frequency domain,

where:

$$
\Delta t_{j} \Delta \omega_{j}=k_{1} k_{2} \text { constant }
$$

The "width" of a function is defined as the interval between which the function is equal to or more than one-half its maximum value: i.e.,

$$
\exp \left\{-b_{j}\left(\Delta t_{j} / 2\right)^{2}\right\}=1 / 2
$$

$$
\exp \left\{-\left(\Delta \omega_{j} / 2\right)^{2} / 4 b_{j}\right\}=1 / 2 \text {. }
$$

From equations 38 and 39 we get,

$$
\begin{aligned}
& b_{j}\left(\Delta t_{j}\right)^{2} / 4=\ln (2) \\
& \left(\Delta \omega_{j}\right)^{2} / 16 b_{j}=\ln (2) .
\end{aligned}
$$

and,

$$
\text { mpute: }
$$

$$
\begin{aligned}
& \left(\Delta t_{j}\right)^{2}\left(\Delta \omega_{j}\right)^{2}=64 .(\ln 2)^{2} \\
& \Delta t_{j} \Delta \omega_{j}=8 \ln 2
\end{aligned}
$$

and

Equation 36 suggests that if we measure the wavelet width in the time domain, we can then estimate the width of the wavelet in the frequency domain. In order not to be influenced by the phase characteristics of the seismic trace, we measure the wavelet width on the envelope of the complex trace. The width is, as defined above, the distance in seconds between 1/2 the maximum amplitude on either side of the envelope peak. The centroid of the frequency spectrum of the wavelet is determined by computing the instantaneous frequency at the peak of the envelope. We now know the centroid and width of the spectrum of each wavelet. Equations 11 and 12 in the frequency shift section 
shows the change of Gaussian shaped spectrum propagating through a constant Q dispersive medium. We have shown that central frequency shifts towards lower frequencies depending on $\mathrm{Q}$ value and travel time. These equations also show that frequency range between $1 / 3$ amplitude points gets shorter. If we compare equation 11 with equation 30 of this section, we see that;

$$
\beta_{j}=1 / 4 b_{j}
$$

and the width of the wavelet in frequency domain is ( from equation 39);

$$
\begin{aligned}
\Delta \omega_{j} & =2 \sqrt{4 b_{j} \cdot \ln 2} \\
\Delta \omega_{j} & =2 \sqrt{\frac{\ln 2}{\beta_{j}}}
\end{aligned}
$$

Equation 12 shows the modification of the spectrum by the $Q$ effect. Since the modification in the log amplitude domain is a linear function with respect to the frequency, amplitudes on the higher frequency side will be suppressed proportionally more than the amplitudes on the lower frequency side. Therefore the bandwidth of the signal will be shortened as well as shifted to lower frequency. To determine the attenuated bandwidth we set the exponential part of equation 12 equal to $\ln (1 / 2)$;

$$
-\beta\left(\omega-\omega_{0}\right)^{2}-\frac{\omega t}{2 Q}=-\ln 2
$$

This can be reduced to a quadratic equation;

$$
\omega^{2}-\left(2 \omega_{0}-t / 2 Q \beta\right) \omega+\omega_{0}^{2}-\ln 2 / \beta=0
$$

The solution of equation 44 will give us the upper and lower $1 / 2$ amplitude limits of the frequency spectrum;

$$
\omega_{u, l}=\left\{\left(2 \omega_{0}-t / 2 Q \beta\right) \pm \sqrt{(2 \omega-t / 2 Q \beta)^{2}-4\left(\omega_{0}^{2}-\ln 2 / \beta\right)}\right\} / 2
$$

We can see from equation 45 that the upper and lower limits will be symmetric around $\omega_{c}=\left(\omega_{0}-t / 4 Q \beta\right)$, which is in accordance with the center frequency predicted by equation 14 . We now simplify the equation 41 . Inside the square root, we have;

$$
\begin{aligned}
& \sqrt{*}=\sqrt{4 \omega_{0}^{2}-2 \omega_{0} / 2 Q \beta+(1 / 2 Q \beta)^{2}-4 \omega_{0}^{2}-4 \ln 2 / \beta} \quad, \text { and } \\
& \sqrt{*}=\sqrt{4 \ln 2 / \beta-2 \omega_{0} / 2 Q \beta+(1 / 2 Q \beta)^{2}}
\end{aligned}
$$

Therefore, equation 45 becomes;

$$
\omega_{u, l}=\left(\omega_{0}-1 / 4 Q \beta\right) \pm \sqrt{4 \ln 2 / \beta-2 \omega_{0} / 2 Q \beta+(1 / 2 Q \beta)^{2}}
$$

Hence, the width of the attenuated wavelet in the frequency domain is; 


$$
\Delta \omega_{0}=2 \sqrt{4 \ln 2 / \beta-2 \omega_{0} / 2 Q \beta+(1 / 2 Q \beta)^{2}}
$$

which is narrower than the original wavelet. This indicates that the width in the time domain will increase.

\section{SOME CORRECTIONS:}

Some of the $\mathrm{Q}$ computations discussed above give instantaneous $\mathrm{Q}$ estimates. It is more reasonable that these estimates are more reliable when they are computed as wavelet attributes at the envelope maxima. Furthermore, they are computed without the removal of the effects of earlier events. They are so-called, "effective Q" estimates. It is generally accepted that the effective $Q$ is the geometric average of intrinsic and extrinsic (scattering) Q;

$$
\frac{1}{Q_{E f f}}=\frac{1}{Q_{\text {Intr }}}+\frac{1}{Q_{\text {Scat }}}
$$

Therefore, in order to compute the local Q, we need to consider the effective Q estimates in two consecutive locations and form the difference;

$$
\frac{1}{Q_{\text {Intr }}(t)}=\frac{1}{Q_{E f f}(t)}-\frac{1}{Q_{E f f}(t-1)}
$$

This will remove all of the previous scattering effects, will leave, if any, scattering effects within the time interval.

This condition will exist on all of the methods, that is we will end up measuring the effective $Q$ within the computation interval. The effective $Q$ may be sufficiently good in intervals not containing many contrasting impedance layers. If, however, the interval contains many highly varying impedance layers, the energy loss due to scattering effects has to be estimated and removed from the intrinsic Q computation. Some of the proposed method of scattering effects are discussed in another report. (Taner, 2002).

\section{CONCLUSIONS:}

In this report we have discussed several $\mathrm{Q}$ computation methods. They include the conventional spectral ratio and the more recent complex trace attribute methods. In some of the methods, such as the second derivative of the trace envelope, we need to determine the computational coefficient, as in the rise time method. The frequency shift method bases the estimate on the assumption of the instantaneous amplitude spectra as Gaussian shape. Other simple shapes have been studied by the investigators. However, simple shapes may have their specific uses, but they may not be suitable for generalization. The bandwidth estimate shown on this report may be more suitable because it does not make any assumption as to the shape of the spectra. A better method, perhaps, is to compute the instantaneous spectra by the Joint Time Frequency analysis and study the frequency shift on the general shape. We can see that this approach basically becomes similar if not the same as the spectral ratio method for constant Q. 
We have presented several methods based on the shape of seismic events; the rise time, seismic attribute based Q estimate, second derivative of envelope and the wavelet width. They all estimate the wavelet width in time and frequency domains by different methods. It is interesting to note that they end with similar results. It becomes obvious that all of the methods are based on the change of the amplitude and phase spectra of propagating wave field over distance. There are a number of methods, some more accurate that others, each can be useful in measuring $\mathrm{Q}$ depending on the objectives of the measurements. In laboratory measurements we may use the most accurate method, most likely the spectral ratio. However, in exploration seismology we could use several levels of accuracy, depending on the exploration objectives. In many instances indication of anomalous absorption is more than sufficient. In reservoir characterization, we may specifically interested in the accurate intrinsic Q.

For all these reasons, we have presented a number of $\mathrm{Q}$ computation methods and will perform them in the QADCOM program. We will run the computation on the real and synthetic data sets and study the degree of accuracies of each method. Since each computation runs with different input and computational complexity, each of the methods according to their range of accuracy and ease of computation, will be suitable for some particular application.

\section{Work Planned for Next Period}

We will test the patchy saturation Q method on data from one or more wells and compare it to other published techniques. The $\mathrm{Q}$ computation from seismic algorithms will be tested on some synthetic data examples and on real data that has been provided by an oil company contributor.

\section{Problems Encountered This Period}

No significant problems have been encountered in our work so far. We are 2-3 weeks behind schedule, primarily because of previous time commitments of the primary researchers. The project will be back on schedule no later than mid 2002. All indications are that the project will progress nicely over the next several months.

\section{REFERENCES:}

Barnes, A. E., 1991, Instantaneous frequency and amplitude at the envelope peak of a constant phase wavelet; Short Note, Geophysics, 56, 1058-1060.

Barnes, A. E, 1993, Instantaneous spectral bandwidth and dominant frequency with applications to seismic reflection data; Geophysics 58, 419-428.

Koehler, F., 1983, Gabor Wavelet Theory; SRC Internal report. 
Morlet, J., Arens E., Fourgeau, E. and Giard D., 1982, Wave propagation and sampling theory-Part 1; Complex signal and scattering in multilayer media. Part I; Geophysics, v.47 no. 2, p. 203-221.

Morlet, J., Arens E., Fourgeau, E. and Giard D., 1982, Wave propagation and sampling theory-Part I1; Sampling theory and Complex waves. Part II; Geophysics.v.47 no. 2, p. 222-236. (* Discussion in GEO-49-09-1562-1563; Reply in GEO-49-09-1564-1564)

Taner, M. T. 1996, $\boldsymbol{Q}$ Computation and Compensation; RSI Technical report

Taner, M. T., Koehler. F. and Sheriff, R. E. , 1979, Complex Seismic Trace Analysis; Geophysics, 44, 1941-1063

Taner, M. T., 2001, Joint Time/Frequency Analysis, Q Quality Factor and Dispersion Computation Using Gabor-Morlet Wavelets or the Gabor-Morlet Transform; RSI Technical Report

Taner, M. T. 2002, Intrinsic Q Computation; RSI Technical report.

Widrow, B. and Stearns S. D.,1985, Adaptive signal processing; Prentice-Hall Book Co. 